\title{
SOME SPECIAL SOLUTIONS OF SELF SIMILAR TYPE IN MHD, OBTAINED BY A SEPARATION METHOD OF VARIABLES
}

\author{
Michel Cessenat ${ }^{1}$ and Philippe Genta ${ }^{1}$
}

\begin{abstract}
We use a method based on a separation of variables for solving a first order partial differential equations system, using a very simple modelling of MHD. The method consists in introducing three unknown variables $\phi_{1}, \phi_{2}, \phi_{3}$ in addition to the time variable $t$ and then in searching a solution which is separated with respect to $\phi_{1}$ and $t$ only. This is allowed by a very simple relation, called a "metric separation equation", which governs the type of solutions with respect to time. The families of solutions for the system of equations thus obtained, correspond to a radial evolution of the fluid. Solving the MHD equations is then reduced to find the transverse component $H_{\Sigma}$ of the magnetic field on the unit sphere $\Sigma$ by solving a non linear partial equation on $\Sigma$. Thus, we generalize ideas of Courant-Friedrichs [7] and of Sedov [11], on dimensional analysis and self-similar solutions.

Résumé. On développe une méthode de séparation de variables pour un système d'équations aux dérivées partielles du premier ordre qui intervient en magnétohydrodynamique dans une modélisation simplifiée. Cette méthode consiste à faire intervenir en plus du temps, de nouvelles variables a priori inconnues $\phi_{1}, \phi_{2}, \phi_{3}$, et à chercher à imposer à la solution du système une séparation des variables vis-à-vis du temps et de $\phi_{1}$ seulement. Ceci est rendu possible à l'aide d'une équation très simple, dite équation de séparation métrique, qui gouverne le type des solutions. On dégage alors des familles de solutions asymptotiques admissibles pour le système d'équations, et qui correspondent à une évolution radiale du fluide. La résolution du système d'équations de la MHD est alors ramenée à déterminer la composante transverse $H_{\Sigma}$ du champ magnétique sur la sphère unité $\Sigma$, par la résolution d'une équation aux dérivées partielles non linéaire sur $\Sigma$. On généralise ainsi des idées de Courant et Friedrichs [7], et de Sedov [11], reliées aux questions d'analyse dimensionnelle et d'autosimilitude.
\end{abstract}

AMS Subject Classification. 76W05.

Received: July 15, 1998.

\section{Modelling in Magnetohydrodynamics}

We study the coupled evolution of a compressible fluid and electromagnetic field using a macroscopic modelling and the following assumptions:

(a) The fluid is a perfect gas, homogeneous and not viscous. The fluid is described by its specific mass $\rho$, its velocity $v$ and its pressure $p$. The fluid equations are obtained by the conservative laws of density, momentum and energy. The fluid is also a perfect medium with respect to electromagnetic properties, isotropic and homogeneous.

Keywords and phrases. Magnetohydrodynamic (MHD), separation of variables, selfsimilar solutions, dimensional analysis.

${ }^{1}$ CEA/DAM, Centre d'Études de Bruyères-le-Châtel, B.P. 12, 91680 Bruyères-le-Châtel, France. 
(b) The equations of evolution of the electromagnetic field are the Maxwell equations with constitutive equations.

\subsection{Electromagnetic modelling}

\subsubsection{Electromagnetic modelling for a medium at rest}

The electromagnetic field is described by the magnetic field $H$, the electric field $E$, the magnetic and electric inductions $B$ and $D$, which satisfy the Maxwell equations (in the International System I.S. of units) ${ }^{1}$ :

$$
\begin{cases}\text { (i) } \quad \frac{\partial B}{\partial t}+\operatorname{rot} E=0, & \frac{\partial D}{\partial t}-\operatorname{rot} H=-j, \\ \text { (ii) } \operatorname{div} B=0, & \operatorname{div} D=\rho_{c},\end{cases}
$$

where $j$ is the current density and $\rho_{c}$ the electric charge density.

In a perfect, isotropic, homogeneous steady medium without time-memory, the following constitutive laws are used:

$$
D=\varepsilon E, \quad B=\mu H, \quad j=\sigma E,
$$

$\varepsilon, \mu, \sigma$ being the permittivity, the permeability and the conductivity of the medium.

\subsubsection{Electromagnetic modelling for a moving medium}

If the evolution of the medium is given by a transformation $u$ in $\mathbb{R}^{3} \times \mathbb{R}, u:(x, t) \in \mathbb{R}^{3} \times \mathbb{R} \rightarrow u(x, t) \in \mathbb{R}^{3} \times \mathbb{R}$, we have to transform the electromagnetic field, Maxwell equations and constitutive relations by $u$. We denote by a prime the transforms of $E, H, B, D, j, \rho_{c}$. When $u$ is a Lorentz transformation, it is well known (see [5,9]) that Maxwell equations are invariant, and constitutive relations are changed into (for a Lorentz transformation with a constant velocity $v$ of the $S^{\prime}$ frame with respect to the reference frame $S$ )

$$
\left\{\begin{array}{lll}
\text { (i) } & D^{\prime}+\frac{1}{c^{2}} v \times H^{\prime}=\varepsilon\left(E^{\prime}+v \times B^{\prime}\right) & \text { (ii) } \quad B^{\prime}-\frac{1}{c^{2}} v \times E^{\prime}=\mu\left(H^{\prime}-v \times D^{\prime}\right) \\
\text { (iii) } & j^{\prime}-\rho_{c}^{\prime} v=\sigma \beta\left(E^{\prime}+v \times B^{\prime}-\frac{E^{\prime} \cdot v}{c^{2}} v\right), & \text { with } \beta=\left(1-\left(v^{2} / c^{2}\right)\right)^{-1 / 2}
\end{array}\right.
$$

In the case of Galilean transformation $(x, t) \rightarrow(x+v t, t)$, the constitutive relations are:

$$
\left\{\begin{array}{l}
B^{\prime}=\mu\left(H^{\prime}-v \times D^{\prime}\right) \\
D^{\prime}=\varepsilon\left(E^{\prime}+v \times B^{\prime}\right) \\
j^{\prime}-\rho_{c}^{\prime} v=\sigma\left(E^{\prime}+v \times B^{\prime}\right)
\end{array}\right.
$$

Finally, the simplified constitutive relations are:

$$
D=\varepsilon E, \quad B=\mu H, \quad j=\sigma(E+v \times B)+\rho_{c} v
$$

with $\varepsilon=\varepsilon_{0}, \mu=\mu_{0},\left(\varepsilon_{0}, \mu_{0}\right.$ the permittivity and the permeability of the free space).

Then $\rho_{c} v$ and $j$ are neglected and these formulas are assumed to be still locally true when $v$ is not a constant.

\footnotetext{
${ }^{1}$ In the whole paper, we use the French notation rot for curl.
} 
1.1.3. Lorentz force density and energy balance law

(a) The action of the electromagnetic field on the fluid is assumed to be given by the Lorentz force density:

$$
F_{L}=\rho_{c} E+j \times B
$$

thus, if the time derivative of the electric induction (or "displacement current") $D$ is neglected in the Maxwell equations, we have:

$$
\left\{\begin{aligned}
F_{L} & =\varepsilon_{0}(\operatorname{div} E) E+\mu_{0}(\operatorname{rot} H) \times H \\
& =\varepsilon_{0}(\operatorname{div} E) E-\mu_{0} \operatorname{grad} \frac{H^{2}}{2}+\mu_{0}(H \cdot \nabla) H,
\end{aligned}\right.
$$

with $(H \cdot \nabla) H_{i}=\sum_{j} H_{j} \frac{\partial H_{i}}{\partial x_{j}}$.

(b) The usual energy balance law with Joule effect is written in the "conservative form"

$$
j \cdot E=-\frac{\partial w_{\mathrm{m}}}{\partial t}-\operatorname{div} S,
$$

with $S=E \times H$ and $w_{\mathrm{m}}=\frac{1}{2}\left(\varepsilon_{0} E^{2}+\mu_{0} H^{2}\right)=\frac{1}{2}(D \cdot E+B \cdot H)$.

\subsubsection{Summary of the electromagnetic equations}

Finally, under the previous assumptions, the Maxwell equations with the constitutive laws are:

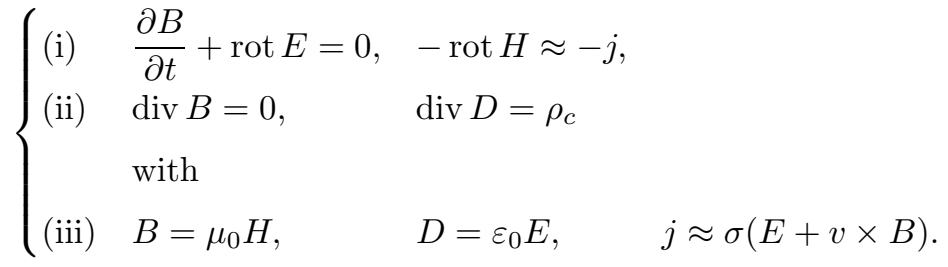

Thus the electric field $E$ is obtained through $H$ by:

$$
E \approx \frac{1}{\sigma} \operatorname{rot} H-\mu_{0} v \times H .
$$

The equation for $H$ is:

$$
\frac{\partial H}{\partial t}-\frac{1}{\sigma \mu_{0}} \Delta H-\operatorname{rot}(v \times H) \approx 0
$$

If we assume, at last, that the fluid conductivity is high so that its corresponding term in the previous equation may be neglected, we obtain "with infinite conduction":

$$
\left\{\begin{array}{l}
\frac{\partial H}{\partial t}-\operatorname{rot}(v \times H)=0, \\
E=-v \times B=-\mu_{0} v \times H .
\end{array}\right.
$$




\section{2. "Hydrodynamics" and fluid modelling}

The fluid evolution in the space $M=\mathbb{R}^{3}$ is given as a function $\phi$ of $a \in \mathbb{R}^{3}$ and time $t$, with regularity properties for every $t$ in the time interval $\left(t_{0}, t_{1}\right)$; the map $a \in \mathbb{R}^{3} \rightarrow \phi(a, t) \in \mathbb{R}^{3}$ must be invertible, continuous and differentiable with its inverse, according to a particle interpretation of the fluid evolution: $a=\left(a_{i}\right)$, $i=1,2,3$, are the Lagrangian coordinates of a fluid particle at time $t=0$ and $x=\left(x_{i}\right)=x_{a, i}(t)=\phi_{i}(a, t)$, $i=1,2,3$ are the Eulerian coordinates of the fluid particle at time $t$.

The field of velocity is obtained from $\phi$ by:

$$
v(x(t), t)=\frac{\mathrm{d}}{\mathrm{d} t} \phi(a, t) \quad \text { i.e. } v(\phi(a, t), t)=\frac{\mathrm{d}}{\mathrm{d} t} \phi(a, t) .
$$

If $f$ is a regular $\left(C^{1}\right)$ function on $\mathbb{R}_{x}^{3} \times \mathbb{R}_{t}$, its particle derivative (or material time derivative) $\mathrm{d} f / \mathrm{d} t$ is the time derivative of $F(t)=f\left(x_{a}(t), t\right)$

$$
\frac{\mathrm{d} f}{\mathrm{~d} t}=\sum_{i} \frac{\partial x_{i}}{\partial t} \cdot \frac{\partial f}{\partial x_{i}}+\frac{\partial f}{\partial t}=v \cdot \operatorname{grad} f+\frac{\partial f}{\partial t} \text { at }(x(t), t) .
$$

The fluid equations (obtained from conservative laws) $\operatorname{are}^{2}$ :

$$
\begin{cases}\text { (i) } \quad & \frac{\mathrm{d} \rho}{\mathrm{d} t}+\rho \operatorname{div} v=0, \\ \text { (ii) } & \rho \frac{\mathrm{d} v}{\mathrm{~d} t}=F_{L}+\operatorname{div} \hat{\sigma}, \\ \text { (iii) } & \rho \frac{\mathrm{d}}{\mathrm{d} t}\left(e+\frac{v^{2}}{2}\right)=j \cdot E+\operatorname{div}(\hat{\sigma} \cdot v)-\operatorname{div} q,\end{cases}
$$

where $\hat{\sigma}$ denote the stress tensor, $e$ the internal energy and $q$ the heat flux.

We assume that:

(i) the stress tensor $\hat{\sigma}$ is reduced to the pressure term $\hat{\sigma}=-p I$ ( $I$ is the identity matrix),

(ii) the heat flux $q$ is null,

(iii) the internal energy $e$ satisfies the law of perfect gas (with $\gamma$ the adiabatic constant):

$$
e=\frac{1}{\gamma-1} \frac{p}{\rho}
$$

The equation (15iii) on internal energy is simplified to:

$$
\rho \frac{\mathrm{d} e}{\mathrm{~d} t}+p \operatorname{div} v=0 .
$$

Thus with (15i), then with (16), we have:

$$
\frac{\mathrm{d} e}{\mathrm{~d} t}-p \frac{1}{\rho^{2}} \frac{\mathrm{d} \rho}{\mathrm{d} t}=0, \text { and } \frac{\mathrm{d} p}{\mathrm{~d} t}+\gamma p \operatorname{div} v=0,
$$

corresponding to the adiabatic law of evolution $p \rho^{-\gamma}=$ constant.

\footnotetext{
${ }^{2}$ See for example [8].
} 


\subsection{Global system of equations}

We simplify and summarize the coupled system of magnetohydrodynamics, as follows. We search for a vector function $U$ of $x$ and $t$ :

$$
U(x, t)=[\rho(x, t), v(x, t), H(x, t), p(x, t)]
$$

with 8 components $U_{j}, j=1, \ldots, 8$,

$$
U_{1}=\rho, \quad U_{1+j}=v_{j}, \quad j=1,2,3, \quad U_{4+j}=H_{j}, j=1,2,3, \quad U_{8}=p,
$$

satisfying the following equations:

$$
\left\{\begin{array}{l}
\frac{\partial \rho}{\partial t}+\operatorname{div}(\rho v)=0, \\
\rho\left(\frac{\partial v}{\partial t}+(v \cdot \nabla) v\right)+\operatorname{grad} p=F_{L}=\mu_{0}(\operatorname{rot} H) \times H, \\
\frac{\partial H}{\partial t}-\operatorname{rot}(v \times H)=0, \text { with } \operatorname{div} H=0, \\
\frac{\partial p}{\partial t}+(v \cdot \nabla) p+\gamma p \operatorname{div} v=0,
\end{array}\right.
$$

or

$$
\left\{\begin{array}{l}
\frac{\mathrm{d} \rho}{\mathrm{d} t}+\rho \operatorname{div} v=0 \\
\rho \frac{\mathrm{d} v}{\mathrm{~d} t}+\operatorname{grad} p=\mu_{0}(\operatorname{rot} H) \times H, \\
\frac{\mathrm{d} H}{\mathrm{~d} t}+H(\operatorname{div} v)-(H \cdot \nabla) v=0, \quad \text { with } \operatorname{div} H=0 \\
\frac{\mathrm{d} p}{\mathrm{~d} t}+\gamma p \operatorname{div} v=0 .
\end{array}\right.
$$

We can write these relations using matrix notations:

$$
\frac{\partial U}{\partial t}+\tilde{A} U=0, \quad \text { or } \frac{\mathrm{d} U}{\mathrm{~d} t}+A U=0 .
$$

Then, with $l=1, \ldots, 8$ and with components $U_{m}(m=1, \ldots, 8)$ of $U$, if $\tilde{A}_{l m}^{j}$ and $A_{l m}^{j}$ are the components of $\tilde{A}$ and $A$, we have:

$$
\frac{\partial U_{l}}{\partial t}+\sum_{j=1,2,3} \sum_{m=1}^{8} \tilde{A}_{l m}^{j} \frac{\partial U_{m}}{\partial x_{j}}=0, \text { or } \frac{\mathrm{d} U_{l}}{\mathrm{~d} t}+\sum_{j=1,2,3} \sum_{m=1}^{8} A_{l m}^{j} \frac{\partial U_{m}}{\partial x_{j}}=0
$$

where

$$
A U=\left(\begin{array}{cccc}
0 & \rho \operatorname{div} & 0 & 0 \\
0 & 0 & \frac{1}{\rho} \mu_{0} a & \frac{1}{\rho} \nabla \\
0 & b & 0 & 0 \\
0 & \gamma p \operatorname{div} & 0 & 0
\end{array}\right) \cdot\left(\begin{array}{c}
\rho \\
v \\
H \\
p
\end{array}\right)
$$


and where

$$
\begin{array}{rlrl}
\rho \operatorname{div} & =\left(\rho \frac{\partial}{\partial x_{1}}, \rho \frac{\partial}{\partial x_{2}}, \rho \frac{\partial}{\partial x_{3}}\right), \quad \nabla & ={ }^{t} \operatorname{div}=\operatorname{grad}, \quad \text { and } a, b \text { the } 3 \times 3 \text { matrix operators } \\
a_{i j} & =H_{j} \partial_{i}-(H \cdot \nabla) \delta_{i j}, \quad b_{i j}=H_{i} \partial_{j}-(H \cdot \nabla) \delta_{i j} .
\end{array}
$$

Using block matrix notations, the matrix operator $A$ is $A=\sum A^{k} \partial_{k}, k=1,2,3$, with

$$
A^{k}=\left(\begin{array}{cccc}
0 & \rho I^{k} & 0 & 0 \\
0 & 0 & \frac{1}{\rho} \mu_{0} a^{k} & \frac{1}{\rho} J^{k} \\
0 & b^{k} & 0 & 0 \\
0 & \gamma p I^{k} & 0 & 0
\end{array}\right)
$$

where $I^{k}$ is an $1 \times 3$ matrix, $J^{k}$ is a $3 \times 1$ matrix, and $a^{k}, b^{k}$ are $3 \times 3$ matrices given by:

$$
\begin{aligned}
\left(I^{k}\right)_{\alpha 1}=\delta_{k a}, \quad\left(J^{k}\right)_{1 \alpha} & =\delta_{k a}, \quad \alpha=1,2,3, \quad \text { thus } J^{k}={ }^{t} I^{k} \\
& \text { and }\left(a^{k}\right)_{i j}=\left(H_{j} \delta_{i k}-H_{k} \delta_{i j}\right), \quad\left(b^{k}\right)_{i j}=\left(H_{i} \delta_{j k}-H_{k} \delta_{i j}\right), \quad i, j=1,2,3, \quad \text { thus } b^{k}={ }^{t} a^{k} .
\end{aligned}
$$

The system of equations (19) is commonly used in MHD, see for example $[1-4,10]$.

\section{Dimensional analysis}

\subsection{Dimensional equations}

At first, we note all these statements are usual in fluid mechanics (Vaschy-Buckingham theorem). We refer also to [11] and to the Russian School (e.g. [3,4], for many studies on Z-pinch).

The state of a physical system at a point $(x, t)\left(x \in \mathbb{R}^{3}\right)$ is given by physical quantities, which basically depend on the chosen units and scale. In the physical systems here considered, relative to mechanics, the basic units are:

$$
\text { length }(L) \text {, time }(T) \text {, mass }(M) \text { and electrical charge }(Q) \text {. }
$$

The physical quantities $v, H, p$ are taken in the international system (I.S.)

$$
[\rho]=L^{-3} M, \quad[v]=L T^{-1}, \quad[H]=L^{-1} T^{-1} Q, \quad[p]=L^{-1} T^{-2} M .
$$

Changing the fundamental units modifies the measured quantities:

$$
\begin{aligned}
& \left(l_{0}, t_{0}, m_{0}, q_{0}\right) \rightarrow\left(l_{1}, t_{1}, m_{1}, q_{1}\right)=\left(\lambda_{1}^{-1} l_{0}, \lambda_{2}^{-1} t_{0}, \lambda_{3}^{-1} m_{0}, \lambda_{4}^{-1} q_{0}\right) \\
& L \rightarrow L^{\prime}=\lambda_{1} L, \quad T \rightarrow T^{\prime}=\lambda_{2} T, \quad M \rightarrow M^{\prime}=\lambda_{3} M, \quad Q \rightarrow Q^{\prime}=\lambda_{4} Q, \\
& \rho^{\prime}=\lambda_{1}^{-3} \lambda_{3} \rho, \quad v^{\prime}=\lambda_{1} \lambda_{2}^{-1} v, \quad H^{\prime}=\lambda_{1}^{-1} \lambda_{2}^{-1} \lambda_{4} H, \quad p^{\prime}=\lambda_{1}^{-1} \lambda_{2}^{-2} \lambda_{3} p .
\end{aligned}
$$

More generally, a physical quantity $A$ with dimensional equation: $[A]=L^{\nu_{1}} T^{\nu_{2}} M^{\nu_{3}} Q^{\nu_{4}}$, with $\nu_{j} \in Z$, is changed into $A^{\prime}=\lambda_{1}^{\nu_{1}} \lambda_{2}^{\nu_{2}} \lambda_{3}^{\nu_{3}} \lambda_{4}^{\nu_{4}} A$. Thus the dimension of $A$ is $\nu=\nu^{A}=\left(\nu_{1}, \nu_{2}, \nu_{3}, \nu_{4}\right) \in Z^{4}$.

The formula of $A^{\prime}$ gives a representation $\Lambda^{\nu}$ of the $\mathbb{R}_{+}^{4}$ group, product of homothetic transformations, in the $\mathbb{R}_{\lambda}$ space

$$
\lambda=\left(\lambda_{1}, \lambda_{2}, \lambda_{3}, \lambda_{4}\right) \in \mathbb{R}_{\lambda}=\mathbb{R}_{+}^{4} \rightarrow \Lambda_{\lambda}^{\nu}=\lambda^{\nu}=\lambda_{1}^{\nu_{1}} \lambda_{2}^{\nu_{2}} \lambda_{3}^{\nu_{3}} \lambda_{4}^{\nu_{4}}
$$


In a Cartesian frame $\left(\left(x_{i}\right) \in \mathbb{R}^{3}\right)$, the unit change of variables is given by the transformation:

$$
x^{\prime}=\lambda_{1} x \quad\left(i . e . x_{j}^{\prime}=\lambda_{1} x_{j}, \quad j=1,2,3\right), \quad t^{\prime}=\lambda_{2} t, \quad \nu\left(x^{j}\right)=\nu(x)=(1,0,0,0), \quad \text { and } \nu(t)=(0,1,0,0) .
$$

The transform of the function $(x, t) \rightarrow A(x, t) \in \mathbb{R}$ associated with the physical quantity $A$ is given by $A^{\prime}\left(x^{\prime}, t^{\prime}\right)=$ $A(x, t)$, with multi-index notations.

For $\lambda=\left(\lambda_{1}, \lambda_{2}\right)$, let $h_{\lambda}$ denote the map $(x, t) \rightarrow \lambda(x, t)=\left(\lambda_{1} x, \lambda_{2} t\right)$ and $A^{\prime}$ the map $\left(x^{\prime}, t^{\prime}\right) \rightarrow A^{\prime}\left(x^{\prime}, t^{\prime}\right)$; we obtain:

$$
A^{\prime} \circ h_{\lambda}=\Lambda_{\lambda}^{\nu} A, \quad \text { or } A^{\prime}=\Lambda_{\lambda}^{\nu} A \circ h_{\lambda}^{-1} .
$$

We have a new representation of the group $\mathbb{R}_{+}^{4}$ in a functional space, as:

$$
A^{\prime}=L_{\lambda}^{\nu}(A)=\Lambda_{\lambda}^{\nu} A \circ h_{\lambda}^{-1},
$$

with

$$
L_{\lambda}^{\nu} L_{\lambda^{\prime}}^{\nu}=L_{\lambda \lambda^{\prime}}^{\nu}, \quad \text { where } \lambda \cdot \lambda^{\prime}=\left(\lambda_{1} \lambda_{1}^{\prime}, \lambda_{2} \lambda_{2}^{\prime}, \lambda_{3} \lambda_{3}^{\prime}, \lambda_{4} \lambda_{4}^{\prime}\right)
$$

This corresponds to the diagram:

$$
\begin{array}{cccc}
(x, t) \in \mathbb{R}^{3} \times \mathbb{R} & \rightarrow A & \rightarrow & A(x, t) \in \mathbb{R} \\
h_{\lambda} \downarrow & L_{\lambda}^{\nu} \downarrow & & \Lambda_{\lambda}^{\nu} \downarrow \\
\left(x^{\prime}, t^{\prime}\right) \in \mathbb{R}^{3} \times \mathbb{R} & \rightarrow \quad A^{\prime} & \rightarrow & A^{\prime}\left(x^{\prime}, t^{\prime}\right) \in \mathbb{R} .
\end{array}
$$

\subsection{Self similar solutions}

For $A$, we choose the components $\left(U_{j}\right), j=1, \ldots, 8$, of $U=(\rho, v, H, p)$ solution of the previous equations of magnetohydrodynamics.

Now we seek solutions of these equations which are invariant under the global change of units. Then we obtain that the solutions (if they exist) have a special "asymptotic behaviour" (with respect to $x$ and $t$ ).

The solution is written as $U_{j}\left(x, t, \mu_{0}\right), j=1, \ldots, 8$, with $\mu_{0}$ the single dimensional constant $\left(\left[\mu_{0}\right]=L M Q^{-2}\right)$.

Changing all units, the solution becomes:

$$
\begin{aligned}
& U_{j}^{\prime}\left(x^{\prime}, t^{\prime} ; \mu_{0}^{\prime}\right)=\Lambda_{j} U_{j}\left(x, t ; \mu_{0}\right), \quad \text { with } \Lambda_{j}=\Lambda^{\nu_{j}}, \quad \nu_{j}=\operatorname{dim} U_{j}, \\
& U_{j}^{\prime}\left(\lambda_{1} x, \lambda_{2} t ; \lambda_{1} \lambda_{3} \lambda_{4}^{-2} \mu_{0}\right)=\Lambda_{j} U_{j}\left(x, t ; \mu_{0}\right), \quad j=1, \ldots, 8 .
\end{aligned}
$$

(a) At first, we prove that there cannot exist a solution that would be invariant under this group of transformations.

If $U_{j}^{\prime}=U_{j}, j=1, \ldots, 8$, we write $x=r \alpha, r=|x|, \alpha=x / r \in S^{2}$ ( $\alpha$ is dimensionless), and

$$
U_{j}\left(\lambda_{1} r, \alpha, \lambda_{2} t ; \lambda_{1} \lambda_{3} \lambda_{4}^{-2} \mu_{0}\right)=\Lambda_{j} U_{j}\left(r, \alpha, t ; \mu_{0}\right)
$$

Then, taking $\lambda_{1} r=1, \lambda_{2} t=1, \lambda_{1} \lambda_{3} \lambda_{4}^{-2} \mu_{0}=1$, and $\zeta^{j}=\nu_{3}^{j}+\left(\nu_{4}^{j} / 2\right)$, we obtain

$$
U_{j}\left(r, \alpha, t ; \mu_{0}\right)=\lambda_{3}^{-\zeta^{j}}\left(r^{\nu_{1}^{j}+\nu_{4}^{j} / 2} t^{\nu_{2}^{j}} \mu_{0}^{-\nu_{4}^{j} / 2}\right) U_{j}(1, \alpha, 1 ; 1)
$$

Finally, $\lambda_{3}$ can be arbitrary and $U_{j}=0$ for $j \neq 2,3,4$, because there is only one dimensional constant $\mu_{0}$ in the equations. 
(b) Now we seek solutions invariant under a subgroup $\mathbb{R}_{+}^{3}$, that is freezing a unit, for example the electrical charge; we have:

$$
U_{j}\left(\lambda_{1} r, \alpha, \lambda_{2} t ; \lambda_{1} \lambda_{3} \mu_{0}\right)=\Lambda_{j} U_{j}\left(r, \alpha, t ; \mu_{0}\right) .
$$

Let $\lambda_{1} r=1, \lambda_{2} t=1, \lambda_{1} \lambda_{3} \mu_{0}=1$. We obtain, with $\psi_{j}(\alpha)=U_{j}(1, \alpha, 1 ; 1)$ a function of $\alpha$ only,

$$
U_{j}\left(r, \alpha, t ; \mu_{0}\right)=\left(\Lambda_{j}\right)^{-1} U_{j}(1, \alpha, 1 ; 1)=r^{\nu_{1}^{j}-\nu_{3}^{j}} t^{\nu_{2}^{j}} \mu_{0}^{\nu_{3}^{j}} \psi_{j}(\alpha) .
$$

We have a natural separation of variables: in general, such a separation with respect to the "natural" variables $(x, t)$ or $(r, \alpha, t)$ is not possible. The idea is now to impose the separation with respect to new unknown variables, as Courant and Friedrichs [7] do it for two variables with two new parameters.

These ideas are similar to ideas of Sedov [11] on П-theorem and self-similarity.

\subsection{Generalized dimensional analysis}

Now let $\tilde{U}_{j}\left(\beta_{1}, \beta_{2}, \psi_{1}, \psi_{2}\right)$ be a solution of the MHD equations, with unknown variables $\beta_{1}, \beta_{2}, \psi_{1}, \psi_{2}$. The quantities $\beta_{1}$ and $\beta_{2}$ have dimensions and are positive, $\psi_{1}$ and $\psi_{2}$ are dimensionless, so that:

$$
\tilde{U}_{j}^{\prime}\left(\lambda_{1} \beta_{1}, \lambda_{2} \beta_{2}, \psi_{1}, \psi_{2}\right)=\lambda_{1}^{\theta_{j}} \lambda_{2}^{\kappa_{j}} \tilde{U}_{j}\left(\beta_{1}, \beta_{2}, \psi_{1}, \psi_{2}\right), \quad \forall \lambda_{1}, \lambda_{2} \in \mathbb{R}^{+},
$$

with real constants $\theta_{j}$ and $\kappa_{j}$ only depending on the dimensions $\nu\left(\tilde{U}_{j}\right)=\tilde{\nu}_{j}$ of $\tilde{U}_{j}$. Assuming that $\left(\tilde{U}_{j}\right)$ is invariant under this change of units, we have:

$$
\tilde{U}_{j}\left(\beta_{1}, \beta_{2}, \psi_{1}, \psi_{2}\right)=\beta_{1}^{\theta_{j}} \beta_{2}^{\kappa_{j}} \tilde{U}_{j}\left(1,1, \psi_{1}, \psi_{2}\right)=\beta_{1}^{\theta_{j}} \beta_{2}^{\kappa_{j}} \chi_{j}\left(\psi_{1}, \psi_{2}\right)
$$

by taking $\lambda_{1} \beta_{1}=1, \lambda_{2} \beta_{2}=1$.

Let us specify the dimensions $\nu\left(\beta_{1}\right), \nu\left(\beta_{2}\right)$ of $\beta_{1}, \beta_{2}$ with respect to the units $L, T, M$ and with respect to coefficients $\theta_{j}$ and $\kappa_{j}$. Let $\nu\left(\beta_{i}\right)=\nu^{i}=\left(\nu_{1}^{i}, \nu_{2}^{i}, \nu_{3}^{i}\right), i=1,2$. Then we have:

$$
\theta_{2}=\theta_{3}=\theta_{4}, \quad \theta_{5}=\theta_{6}=\theta_{7}, \quad \text { and } \kappa_{2}=\kappa_{3}=\kappa_{4}, \quad \kappa_{5}=\kappa_{6}=\kappa_{7} .
$$

Notice that $H\left(\mu_{0} / p\right)^{1 / 2}$ and $v(\rho / p)^{1 / 2}$ are dimensionless and $[H]=[p]^{1 / 2}$, thus:

$$
\theta_{5}=\frac{1}{2} \theta_{8}, \quad \frac{\theta_{1}}{2}+\theta_{2}-\frac{\theta_{8}}{2}=0, \quad \text { and } \kappa_{5}=\frac{1}{2} \kappa_{8}, \quad \frac{\kappa_{1}}{2}+\kappa_{2}-\frac{\kappa_{8}}{2}=0 .
$$

Let

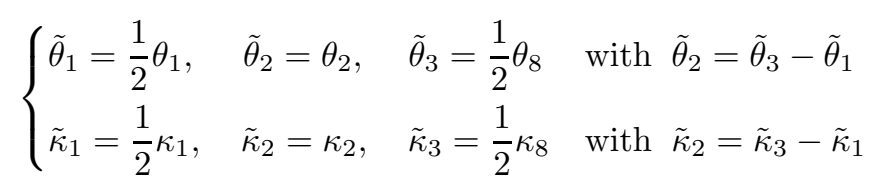

and also

$$
\begin{cases}\theta=\tilde{\theta}_{3}, & \theta_{0}=\tilde{\theta}_{1} \quad \text { thus: } \tilde{\theta}_{2}=\theta-\theta_{0} \\ \kappa=\tilde{\kappa}_{3}, & \kappa_{0}=\tilde{\kappa}_{1} \quad \text { thus: } \quad \tilde{\kappa}_{2}=\kappa-\kappa_{0} .\end{cases}
$$


Then the dimensional equations for $\rho$ and $p$ are:

$$
\left\{\begin{array}{l}
\nu(\rho)=(-3,0,1)=2 \tilde{\theta}_{1}\left(\nu_{1}^{1}, \nu_{2}^{1}, \nu_{3}^{1}\right)+2 \tilde{\kappa}_{1}\left(\nu_{1}^{2}, \nu_{2}^{2}, \nu_{3}^{2}\right) \\
\nu(p)=(-1,-2,1)=2 \tilde{\theta}_{3}\left(\nu_{1}^{1}, \nu_{2}^{1}, \nu_{3}^{1}\right)+2 \tilde{\kappa}_{3}\left(\nu_{1}^{2}, \nu_{2}^{2}, \nu_{3}^{2}\right) .
\end{array}\right.
$$

It is a system of 6 equations and 6 unknowns $\nu_{j}^{i}(i=1,2 ; j=1, \ldots, 3)$, with $\tilde{\theta}_{j}$ and $\tilde{\kappa}_{j}$ as data. This system has a unique solution if

$$
\Delta \equiv \tilde{\theta}_{1} \tilde{\kappa}_{3}-\tilde{\theta}_{3} \tilde{\kappa}_{1}=\theta_{0} \kappa-\theta \kappa_{0} \neq 0
$$

and this solution is:

$$
\nu\left(\beta_{1}\right)=\frac{1}{2 \Delta}\left(\kappa_{0}-3 \kappa, 2 \kappa_{0}, \kappa-\kappa_{0}\right), \quad \nu\left(\beta_{2}\right)=\frac{1}{2 \Delta}\left(-\theta_{0}+3 \theta,-2 \theta_{0},-\theta+\theta_{0}\right) .
$$

\section{Differential GeOMETRY ANALYSiS}

\subsection{The method}

The basic idea is to seek a solution $\tilde{U}_{m}$ of the MHD equations, that is invariant with respect to unit changes

of quantities denoted by $\beta_{1}, \tilde{\beta}_{1}$ and that would be a product of factors with respect to new unknown variables $\tau, \phi_{1}, \phi_{2}, \phi_{3}$ as:

$$
\tilde{U}_{m}\left(\tau, \phi_{1}, \phi_{2}, \phi_{3}\right)=\beta_{1}^{\theta_{m}}(\tau) \tilde{\beta}_{1}^{\kappa_{m}}\left(\phi_{1}\right) W_{m}\left(\phi_{2}, \phi_{3}\right), \quad m=1, \ldots, 8 ;
$$

$\nu_{\beta_{1}}\left(\tilde{U}_{m}\right)=\theta_{m}, \nu_{\tilde{\beta}_{1}}\left(\tilde{U}_{m}\right)=\kappa_{m}$ are the two dimensions of $\tilde{U}_{m}$.

We also have to find the change of variables from the usual coordinates of time and space to $(\tau, \phi)$.

In Eulerian coordinates, $x$ and $t$ are not independent variables, thus we have to introduce a new time variable, denoted by $t^{\prime}$, and to obtain the transformation $\Theta:\left(t^{\prime}, x\right) \rightarrow(\tau, \phi)$ through its Jacobian matrix $J$.

We will choose $\tau=t^{\prime}$ (the change of notation is only a question of writing partial derivatives) and then we choose $\phi_{2}$ and $\phi_{3}$ independent of $t^{\prime}$. The main point is that the velocity of the fluid particle is part of the unknowns $\left(U_{m}\right)$, and thus, must satisfy the separation of variables, as is the formula (45):

$$
\tilde{v}_{i}(\tau, \phi)=\beta_{1}^{\theta_{1+i}}(\tau) \tilde{\beta}_{1}^{\kappa_{1+i}}\left(\phi_{1}\right) W_{1+i}\left(\phi_{2}, \phi_{3}\right), \quad i=1,2,3 .
$$

Now the change of coordinates will be obtained through the differential forms:

$$
\mathrm{d} x_{i}=v_{i} \mathrm{~d} \tau+\sum_{j=1,2,3} a_{i j} \mathrm{~d} \phi_{j}, \quad i=1,2,3, \quad \mathrm{~d} t^{\prime}=\mathrm{d} \tau,
$$

with factors $a_{i j}$ to be defined later. In the whole space $\mathbb{R}_{t} \times \mathbb{R}_{x}^{3}$, we define the operator:

$$
\frac{\mathrm{d}}{\mathrm{d} t^{\prime}}=\sum_{j} v_{j} \frac{\partial}{\partial x_{j}}+\frac{\partial}{\partial t^{\prime}}
$$

also written $\left(\begin{array}{l}v \\ 1\end{array}\right)$ in the basis $\partial / \partial x_{j}, \partial / \partial t^{\prime}$. We calculate $\mathrm{d} \phi_{i} / \mathrm{d} t^{\prime}$ when $i=1,2,3$ :

$$
\left\langle\mathrm{d} \phi_{i}, \frac{\mathrm{d}}{\mathrm{d} t^{\prime}}\right\rangle=\left\langle\sum_{j} \frac{\partial \phi_{i}}{\partial x_{j}} \mathrm{~d} x_{j}+\frac{\partial \phi_{i}}{\partial t^{\prime}} \mathrm{d} t^{\prime}, \sum_{k} v_{k} \frac{\partial}{\partial x_{k}}+\frac{\partial}{\partial t^{\prime}}\right\rangle=\sum_{j} \frac{\partial \phi_{i}}{\partial x_{j}} v_{j}+\frac{\partial \phi_{i}}{\partial t^{\prime}}=0 .
$$


Moreover, we assume that:

$$
\frac{\partial \phi_{2}}{\partial t^{\prime}}=\frac{\partial \phi_{3}}{\partial t^{\prime}}=0
$$

We have the basic formulas of transformation

$$
\begin{aligned}
& J\left(\frac{\mathrm{d}}{\mathrm{d} t^{\prime}}\right)=J\left(\begin{array}{l}
v \\
1
\end{array}\right)=\sum_{i=1}^{3}\left\langle\mathrm{~d} \phi_{i},\left(\begin{array}{l}
v \\
1
\end{array}\right)\right\rangle \frac{\partial}{\partial \phi_{i}}+\left\langle\mathrm{d} \tau,\left(\begin{array}{l}
v \\
1
\end{array}\right)\right\rangle \frac{\partial}{\partial \tau}=\frac{\partial}{\partial \tau} \\
& J\left(\sum v_{i} \frac{\partial}{\partial x_{i}}\right)=J\left(\begin{array}{l}
v \\
0
\end{array}\right)=-\frac{\partial \phi_{1}}{\partial t^{\prime}} \frac{\partial}{\partial \phi_{1}} .
\end{aligned}
$$

The velocity of the fluid particle must be of the form, with separate variables:

$$
v=\frac{\alpha_{1}}{\kappa_{1}^{\prime}} N_{0} w, \quad \text { i.e., } \quad v_{i}=\frac{\alpha_{1}}{\kappa_{1}^{\prime}} N_{0} w_{i}, \quad i=1,2,3, \quad \text { with } \frac{\alpha_{1}}{\kappa_{1}^{\prime}} w_{i}=W_{i+1}, \quad \alpha_{1} \text { constant }
$$

with

$$
N_{0}\left(\tau, \phi_{1}\right)=\beta_{1}(\tau)^{\theta_{1}^{\prime}} \tilde{\beta}_{1}\left(\phi_{1}\right)^{\kappa_{1}^{\prime}}, \quad \text { with } \vartheta_{1}^{\prime}=\tilde{\theta}_{2}=\theta-\theta_{0}, \quad \kappa_{1}^{\prime}=\tilde{\kappa}_{2}=\kappa-\kappa_{0} .
$$

Let

$$
\Gamma=\frac{\beta_{1}}{\beta_{1}^{\prime}}, \quad \tilde{\Gamma}=\frac{\tilde{\beta}_{1}}{\tilde{\beta}_{1}^{\prime}}, \quad G=\frac{\Gamma}{\tilde{\Gamma}}, \quad \text { with } \beta_{1}^{\prime}, \tilde{\beta}_{1}^{\prime}, \tilde{\beta}_{1} \neq 0
$$

Then

$$
\frac{\partial N_{0}}{\partial \tau}=\theta_{1}^{\prime} \frac{1}{\Gamma} N_{0}, \quad \frac{\partial N_{0}}{\partial \phi_{1}}=\kappa_{1}^{\prime} \frac{1}{\tilde{\Gamma}} N_{0}
$$

Relations (46) must correspond to closed differential forms, thus the Schwarz conditions $\frac{\partial a_{i j}}{\partial \tau}=\frac{\partial v_{i}}{\partial \phi_{j}}, i, j=1,2,3$, must be satisfied, thus factors $a_{i j}$ in (46) are so that

$$
\begin{aligned}
& a_{i 1}=\frac{\kappa_{1}^{\prime}}{\alpha_{1}} G v_{i}=\frac{\kappa_{1}^{\prime}}{\alpha_{1}} \frac{\Gamma}{\tilde{\Gamma}} v_{i}=G N_{0} w_{i}, \quad i=1,2,3, \\
& a_{i j}=\frac{\Gamma}{\kappa_{1}^{\prime}} N_{0} w_{i, j}, \quad i=1,2,3, \quad j=2,3, \quad w_{i, j}=\frac{\partial w_{i}}{\partial \phi_{j}} .
\end{aligned}
$$

Then (46) can be written as:

$$
\left\{\begin{array}{l}
\mathrm{d} x_{i}=\frac{\alpha_{1}}{\kappa_{1}^{\prime}} N_{0} w_{i} \mathrm{~d} \tau+G N_{0} w_{i} \mathrm{~d} \phi_{1}+\frac{1}{\kappa_{1}^{\prime}} \Gamma N_{0}\left(\frac{\partial w_{i}}{\partial \phi_{2}} \mathrm{~d} \phi_{2}+\frac{\partial w_{i}}{\partial \phi_{3}} \mathrm{~d} \phi_{3}\right), \quad i=1,2,3 \\
\mathrm{~d} t^{\prime}=\mathrm{d} \tau .
\end{array}\right.
$$

Obviously, the differential forms $\mathrm{d} x_{i}$ are closed forms if and only if:

$$
\frac{\partial \Gamma}{\partial \tau}=\alpha_{1}-\theta_{1}^{\prime}
$$

This is called the Metric Separation Equation (MSE). Thus we have the transformation formulas $(\tau, \phi) \rightarrow\left(t^{\prime}, x\right)$

$$
x_{i}=\frac{1}{\kappa_{1}^{\prime}} \Gamma(\tau) N_{0}\left(\tau, \phi_{1}\right) w_{i}\left(\phi_{2}, \phi_{3}\right), \quad i=1,2,3 \quad \text { and } t^{\prime}=\tau .
$$




\subsection{Path of the fluid particle}

Now the path of the fluid particle which is at a point $a \in \mathbb{R}^{3}$ at initial time $t=0$, is given by the function:

$$
t \in \mathbb{R} \rightarrow X_{a}(t)=\left(t, x_{a}(t)\right) \in \mathbb{R} \times \mathbb{R}^{3} \text { with } x_{a}(0)=a .
$$

Now, at point $X_{a}(t)$, we have $t=t^{\prime}=\tau, x_{a, i}(t)=x_{i}(\tau, \phi), i=1,2,3$. Thus

$$
x_{a, i}(t)=\frac{1}{\kappa_{1}^{\prime}} \Gamma(t) N_{0}\left(\tau, \phi_{1}\right) w_{i}\left(\phi_{2}, \phi_{3}\right), \quad i=1,2,3 .
$$

Therefore

$$
\left\{\begin{array}{l}
x_{a, i}(0)=a_{i}=\frac{1}{\kappa_{1}^{\prime}} \Gamma(0) N_{0}\left(0, \phi_{1}\right) w_{i}\left(\phi_{2}, \phi_{3}\right)=\frac{1}{\kappa_{1}^{\prime}} C \tilde{\beta}_{1}^{\kappa_{1}^{\prime}}\left(\phi_{1}\right) w_{i}\left(\phi_{2}, \phi_{3}\right) \\
\text { with } C=\left(\Gamma \beta_{1}^{\theta_{1}^{\prime}}\right)_{\tau=0} \text { constant. }
\end{array}\right.
$$

Let $M(t)=\Gamma(t) \beta_{1}^{\theta_{1}^{\prime}}(t) / \Gamma(0) \beta_{1}^{\theta_{1}^{\prime}}(0)$. From (60), the evolution is given by

$$
x_{a, i}(t)=M(t) a_{i} \quad \text { i.e. } x_{a}(t)=M(t) a,
$$

and the velocity of the fluid particle is:

$$
\left\{\begin{aligned}
v_{a, i}(t) & =\frac{\mathrm{d}}{\mathrm{d} t} x_{a, i}(t)=\frac{1}{\kappa_{1}^{\prime}}\left(\Gamma^{\prime} N_{0}+\Gamma \frac{\theta_{1}^{\prime}}{\Gamma} N_{0}\right) w_{i}=\frac{\alpha_{1}}{\kappa_{1}^{\prime}} N_{0}\left(t, \phi_{1}\right) w_{i}\left(\phi_{2}, \phi_{3}\right) \\
& =v_{i}(\phi, \tau) .
\end{aligned}\right.
$$

Note that it is also the partial derivative of the position with respect to $\tau$ :

$$
v_{a}(t)=\frac{\mathrm{d}}{\mathrm{d} t} x_{a}(t)=v(\phi, \tau)=\frac{\partial x}{\partial \tau}(\phi, \tau) .
$$

Then (62) implies

$$
v_{i}=\frac{\alpha_{1}}{\Gamma(t)} x_{i}, \quad \text { i.e., } \quad v\left(x_{a}(t), t\right)=\frac{\mathrm{d} x_{a}(t)}{\mathrm{d} t}=\frac{\alpha_{1}}{\Gamma(t)} x_{a}(t) .
$$

Thus the flow of the fluid particle is radial. Note that the field of velocity is curl free:

$$
\operatorname{rot} v=\frac{\alpha_{1}}{\Gamma(t)} \operatorname{rot} x=0
$$

From these results and the relation

$$
\frac{\partial \phi_{1}}{\partial t^{\prime}}=-\frac{\alpha_{1}}{\kappa_{1}^{\prime}} G^{-1}=-\frac{\alpha_{1}}{\kappa_{1}^{\prime}} \frac{\tilde{\Gamma}}{\Gamma}
$$

we can prove (using the Euler equation of homogeneous functions) that $\tilde{\beta}_{1}$ is

$$
\tilde{\beta}_{1}\left(\phi_{1}\right)=\tilde{C}_{1}\left(\beta_{1}^{-\alpha_{1}} r\right)^{1 / \kappa_{1}^{\prime}}, \quad \text { or } \tilde{\beta}_{1}^{\kappa_{1}^{\prime}}\left(\phi_{1}\right)=\tilde{C} \beta_{1}^{-\alpha_{1}} r, \quad \tilde{C}_{1}, \tilde{C} \text { constant, } \tilde{C}_{1}^{\kappa^{\prime}}=\tilde{C},
$$


and we get that $\phi_{1}\left(x_{a}(t), t\right)$ only depends on $a=\left(a_{1}, a_{2}, a_{3}\right)$ :

$$
\begin{aligned}
& \tilde{\beta}_{1}^{\kappa_{1}^{\prime}}\left(\phi_{1}\left(x_{a}(t), t^{\prime}\right)\right)=\tilde{C} \beta_{1}^{-\alpha_{1}}\left(t^{\prime}\right) r_{a}(t)=\tilde{C} \beta_{1}^{-\alpha_{1}}\left(t^{\prime}\right) M(t)|a|=\tilde{C} \beta_{1}^{-\alpha_{1}}(0) \frac{M(t)}{M\left(t^{\prime}\right)}|a|, \\
& \tilde{\beta}_{1}^{\kappa_{1}^{\prime}}\left(\phi_{1}\left(x_{a}\left(t^{\prime}\right), t^{\prime}\right)\right)=\tilde{C} \beta_{1}^{-\alpha_{1}}(0)|a|, \quad \tilde{\beta}_{1}^{\kappa_{1}^{\prime}}\left(\phi_{1}\left(x_{a}(t), t^{\prime}\right)\right)=\frac{M(t)}{M\left(t^{\prime}\right)} \tilde{\beta}_{1}^{\kappa_{1}^{\prime}}\left(\phi_{1}\left(x_{a}\left(t^{\prime}\right), t^{\prime}\right)\right) .
\end{aligned}
$$

Notice also, [from (59)], that the directions of $x$ and $w$ are identical, and more precisely, using $(59,60,66)$, that there exists a constant $C_{0}$ (independent of $(\tau, \phi)$ )

$$
C_{0}=\frac{1}{\kappa_{1}^{\prime}} \tilde{C} \beta_{1}^{\theta_{1}^{\prime}-\alpha_{1}}(0) \Gamma(0),
$$

such that

$$
\frac{x_{i}}{|x|}=C_{0} w_{i}, \quad \text { thus }|w|=\frac{1}{C_{0}}, \quad \text { and } \frac{x}{|x|}=\tilde{w}\left(\phi_{2}, \phi_{3}\right)=\frac{w}{|w|} .
$$

Therefore $\left(\phi_{2}, \phi_{3}\right)$ may be chosen as spherical coordinates.

\subsection{Some general results}

To solve the system of equations given by a particular modelling of MHD, we need the value of $\beta_{1}, \tilde{\beta}_{1}$ through the MSE. We need also differential operators such as the partial derivatives $\partial / \partial t, \partial / \partial x_{i}, \partial^{2} / \partial^{2} x_{i}$, grad, div, rot, v. grad, $\Delta$. So we write down here the most useful formulas and we calculate also some interesting matrices deduced from $J$.

3.3.1. Solutions of the "metric separation equation" (MSE)

Through the MSE, we will get $\beta_{1}(\tau)$. We recall [see (57)]

$$
\frac{\mathrm{d} \Gamma}{\mathrm{d} \tau}=\alpha_{1}-\theta_{1}^{\prime}, \quad \text { with } \Gamma=\frac{\beta_{1}}{\beta_{1}^{\prime}} .
$$

We have two cases.

(i) $\alpha_{1}-\theta_{1}^{\prime} \neq 0$ : Then we can define $\lambda_{1}=\left(\alpha_{1}-\theta_{1}^{\prime}\right)^{-1}$ and:

$$
\beta_{1}(\tau)=C_{1}\left(\frac{\tau+\tau_{0}}{\tau_{0}}\right)^{\lambda_{1}} \quad \text { with } \beta_{0}(0)=C_{1}>0 .
$$

The evolution operator of the fluid particle is:

$$
M(t)=\left(\frac{\tau+\tau_{0}}{\tau_{0}}\right)^{\alpha_{1} /\left(\alpha_{1}-\theta_{1}^{\prime}\right)} .
$$

(ii) $\alpha_{1}-\theta_{1}^{\prime}=0$ :

$$
\beta_{1}(\tau)=C_{1} \exp \left(\tau / \tau_{1}\right) \text { with } C_{1}=\beta_{1}(0)>0
$$

and then

$$
M(t)=\exp \left(\theta_{1}^{\prime} t / \tau_{1}\right) .
$$

Now, we have $\tilde{\beta}_{1}$ from $\beta_{1}[$ see $(65,66)]$. 
3.3.2. Formulation of the MHD equations using the variables $(\phi, \tau)$

Let $\Theta$ be the change of coordinates $\left(x, t^{\prime}\right) \rightarrow(\phi, \tau)=\left(\phi_{1}, \phi_{2}, \phi_{3}, \tau\right)$. We define:

$$
\tilde{U}(\phi, \tau)=U\left(\Theta^{-1}(\phi, \tau)\right), \quad \tilde{X}_{a}(t)=\Theta\left(X_{a}(t)\right)
$$

thus $U\left(X_{a}(t)\right)=\tilde{U}\left(\Theta\left(X_{a}(t)\right)\right)=\tilde{U}\left(\tilde{X}_{a}(t)\right)$. Then we can prove that

$$
\frac{\mathrm{d}}{\mathrm{d} t} U\left(X_{a}(t)\right)=\frac{\mathrm{d}}{\mathrm{d} t} \tilde{U}\left(\tilde{X}_{a}(t)\right)=\left.\partial_{0} \tilde{U}\right|_{\tilde{X}_{a}(t)}=\left.\frac{\partial \tilde{U}}{\partial \tau}\right|_{\tilde{X}_{a}(t)} .
$$

The MHD equations of Section 1.3 are transformed into

$$
\left.\left[\frac{\partial}{\partial \tau} \tilde{U}_{l}+\sum_{j=1}^{3} \sum_{m} B_{l m}^{j} \frac{\partial \tilde{U}_{m}}{\partial \phi_{j}}\right]\right|_{\tilde{X}_{a}(t)}=\tilde{F}_{l}\left(\tilde{X}_{a}(t)\right)
$$

with

$$
B_{l m}^{j}\left(\tilde{X}_{a}(t)\right)=\sum_{i=1}^{3} A_{l m}^{i}\left(X_{a}(t)\right) \frac{\partial \phi_{j}}{\partial x_{i}}\left(X_{a}(t)\right)=\left(\tilde{J}\left(A_{l m}\right)\right)_{j}
$$

and thus, using $(45,53)$ with $N_{m}=\beta_{1}^{\theta_{m}} \tilde{\beta}_{1}^{\kappa_{m}}$, the MHD equations are

$$
\frac{1}{\Gamma} \theta_{l} N_{l} W_{l}+\sum_{m} \frac{1}{\tilde{\Gamma}} \kappa_{m} N_{m} B_{l m}^{1} W_{m}+\sum_{j=2,3} \sum_{m} B_{l m}^{j} N_{m} \frac{\partial W_{m}}{\partial \phi_{j}}=\tilde{F}_{l}, \quad l=1, \ldots, 8 .
$$

\subsubsection{Jacobian matrix and scale factors}

\section{$\underline{\text { Transformation in } \mathbb{R}^{4}}$}

The Jacobian matrix $J$ of the change of variables $\Theta:\left(x, t^{\prime}\right) \rightarrow(\phi, \tau)$ is given by the dyadic expression, with $\phi_{0}=\tau, x_{0}=t^{\prime}$ :

$$
J=\sum_{i=0}^{3} \frac{\partial}{\partial \phi_{i}} \otimes \mathrm{d} \phi_{i}=\sum_{i, j=0}^{3} \frac{\partial \phi_{i}}{\partial x_{j}} \frac{\partial}{\partial \phi_{i}} \otimes \mathrm{d} x_{j}
$$

The transform of the vector $A=\sum_{j=0, \ldots, 3} A_{i} \frac{\partial}{\partial x_{j}}$ is $J(A)=\sum_{i}(\tilde{J}(A))_{i} \frac{\partial}{\partial \phi_{i}}$ with:

$$
(\tilde{J}(A))_{i}=\sum_{j} \frac{\partial \phi_{i}}{\partial x_{j}} A_{j}, \quad \text { with } \tilde{J}=\left(\tilde{J}_{i, j}\right)=\left(\frac{\partial \phi_{i}}{\partial x_{j}}\right)
$$

Then the inverse of the Jacobian matrix $J$ is given by:

$$
J^{-1}=\sum_{j=0}^{3} \frac{\partial}{\partial x_{j}} \otimes \mathrm{d} x_{j}=\sum_{i, j=0}^{3} \frac{\partial x_{j}}{\partial \phi_{i}} \frac{\partial}{\partial x_{j}} \otimes \mathrm{d} \phi_{i} .
$$


Let $B$ denote a tangent vector field in the $\phi$ variables:

$$
B=\sum_{i=0}^{3} B_{i} \frac{\partial}{\partial \phi_{i}}=B_{0} \frac{\partial}{\partial \tau}+\sum_{i=1}^{3} B_{i} \frac{\partial}{\partial \phi_{i}}
$$

The transform $A$ of $B$ by the change of coordinates $\Theta^{-1}$ is

$$
A=\sum_{j=0}^{3} A_{j} \frac{\partial}{\partial x_{j}}=J^{-1} B=\sum_{j, i=0}^{3} B_{i} \frac{\partial x_{j}}{\partial \phi_{i}} \frac{\partial}{\partial x_{j}}, \text { thus } A_{j}=\sum_{i=0}^{3} \frac{\partial x_{j}}{\partial \phi_{i}} B_{i} .
$$

To change the components $\left(B_{i}\right)$ to $\left(A_{j}\right)$, with $(A)=\tilde{J}^{-1}(B)$, use $\tilde{J}^{-1}$ given by:

$$
\tilde{J}^{-1}=\left(\begin{array}{cccc}
\frac{\partial t^{\prime}}{\partial \tau} & \frac{\partial t^{\prime}}{\partial \phi_{1}} & \frac{\partial t^{\prime}}{\partial \phi_{2}} & \frac{\partial t^{\prime}}{\partial \phi_{3}} \\
\frac{\partial x_{1}}{\partial \tau} & \frac{\partial x_{1}}{\partial \phi_{1}} & \frac{\partial x_{1}}{\partial \phi_{2}} & \frac{\partial x_{1}}{\partial \phi_{3}} \\
\cdot & \cdot & \cdot & \cdot \\
\cdot & \cdot & \cdot & \frac{\partial x_{3}}{\partial \phi_{3}}
\end{array}\right)=\left(\begin{array}{ccccc}
1 & 0 & 0 & 0 \\
\frac{\alpha_{1}}{\kappa_{1}^{\prime}} N_{0} w_{1} & G N_{0} w_{1} & \frac{1}{\kappa_{1}^{\prime}} \Gamma N_{0} \frac{\partial w_{1}}{\partial \phi_{2}} & \frac{1}{\kappa_{1}^{\prime}} \Gamma N_{0} \frac{\partial w_{1}}{\partial \phi_{3}} \\
\frac{\alpha_{1}}{\kappa_{1}^{\prime}} N_{0} w_{2} & G N_{0} w_{2} & \frac{1}{\kappa_{1}^{\prime}} \Gamma N_{0} \frac{\partial w_{2}}{\partial \phi_{2}} & \frac{1}{\kappa_{1}^{\prime}} \Gamma N_{0} \frac{\partial w_{2}}{\partial \phi_{3}} \\
\frac{\alpha_{1}}{\kappa_{1}^{\prime}} N_{0} w_{3} & G N_{0} w_{3} & \frac{1}{\kappa_{1}^{\prime}} \Gamma N_{0} \frac{\partial w_{3}}{\partial \phi_{2}} & \frac{1}{\kappa_{1}^{\prime}} \Gamma N_{0} \frac{\partial w_{3}}{\partial \phi_{3}}
\end{array}\right)
$$

\section{Transformation in $\mathbb{R}^{3}$}

Let $B=\sum_{i=1}^{3} B_{i} \frac{\partial}{\partial \phi_{i}}$ and $A$ be given by $A=\sum_{j=1}^{3} A_{j} \frac{\partial}{\partial x_{j}}$ (thus with $B_{0}=0, A_{0}=0$ ) with

$$
\left(\begin{array}{l}
A_{1} \\
A_{2} \\
A_{3}
\end{array}\right)=J_{0}^{-1}\left(\begin{array}{l}
B_{1} \\
B_{2} \\
B_{3}
\end{array}\right)=\left(\begin{array}{lll}
G N_{0} w_{1} & \frac{1}{\kappa_{1}^{\prime}} \Gamma N_{0} \frac{\partial w_{1}}{\partial \phi_{2}} & \frac{1}{\kappa_{1}^{\prime}} \Gamma N_{0} \frac{\partial w_{1}}{\partial \phi_{3}} \\
G N_{0} w_{2} & \frac{1}{\kappa_{1}^{\prime}} \Gamma N_{0} \frac{\partial w_{2}}{\partial \phi_{2}} & \frac{1}{\kappa_{1}^{\prime}} \Gamma N_{0} \frac{\partial w_{2}}{\partial \phi_{3}} \\
G N_{0} w_{3} & \frac{1}{\kappa_{1}^{\prime}} \Gamma N_{0} \frac{\partial w_{3}}{\partial \phi_{2}} & \frac{1}{\kappa_{1}^{\prime}} \Gamma N_{0} \frac{\partial w_{3}}{\partial \phi_{3}}
\end{array}\right)\left(\begin{array}{l}
B_{1} \\
B_{2} \\
B_{3}
\end{array}\right)
$$

Let $\tilde{B}$ denote the vector with components:

$$
\tilde{B}_{1}=G N_{0} B_{1}, \quad \tilde{B}_{2}=\frac{1}{\kappa_{1}^{\prime}} \Gamma N_{0} B_{2}, \quad \tilde{B}_{3}=\frac{1}{\kappa_{1}^{\prime}} \Gamma N_{0} B_{3}
$$

Let $J_{1}$ be this transformation from $\tilde{B}$ to $A$ :

$$
\left(\begin{array}{l}
A_{1} \\
A_{2} \\
A_{3}
\end{array}\right)=J_{1}\left(\begin{array}{l}
\tilde{B}_{1} \\
\tilde{B}_{2} \\
\tilde{B}_{3}
\end{array}\right)=\left(\begin{array}{lll}
w_{1} & \frac{\partial w_{1}}{\partial \phi_{2}} & \frac{\partial w_{1}}{\partial \phi_{3}} \\
w_{2} & \frac{\partial w_{2}}{\partial \phi_{2}} & \frac{\partial w_{2}}{\partial \phi_{3}} \\
w_{3} & \frac{\partial w_{3}}{\partial \phi_{2}} & \frac{\partial w_{3}}{\partial \phi_{3}}
\end{array}\right)\left(\begin{array}{l}
\tilde{B}_{1} \\
\tilde{B}_{2} \\
\tilde{B}_{3}
\end{array}\right)
$$


Now, if we define the scale factor matrix $\Lambda$ by

$$
\Lambda=\left(\begin{array}{ccc}
\frac{1}{G N_{0}} & 0 & 0 \\
0 & \kappa_{1}^{\prime} \frac{1}{\Gamma N_{0}} & 0 \\
0 & 0 & \kappa_{1}^{\prime} \frac{1}{\Gamma N_{0}}
\end{array}\right)
$$

then the matrix $J_{0}^{-1}$ defined by (85) is given by

$$
J_{0}^{-1}=J_{1} \Lambda^{-1}
$$

Let $J_{1}^{-1}$ denote the dimensionless matrix:

$$
J_{1}^{-1}=\left(\begin{array}{lll}
\eta_{1} & \eta_{2} & \eta_{3} \\
\delta_{1} & \delta_{2} & \delta_{3} \\
\varepsilon_{1} & \varepsilon_{2} & \varepsilon_{3}
\end{array}\right)
$$

Then the matrix $J_{0}$ [the inverse of $J_{0}^{-1}$ given by (85)] is $J_{0}=\Lambda J_{1}^{-1}$, and the coefficients of $J_{0}$ are:

$$
\frac{\partial \phi_{1}}{\partial x_{j}}=\frac{1}{G N_{0}} \eta_{j}, \quad \frac{\partial \phi_{2}}{\partial x_{j}}=\kappa_{1}^{\prime} \frac{1}{\Gamma N_{0}} \delta_{j}, \quad \frac{\partial \phi_{3}}{\partial x_{j}}=\kappa_{1}^{\prime} \frac{1}{\Gamma N_{0}} \varepsilon_{j}, \quad j=1,2,3 .
$$

The first order derivatives of $U_{l}$ are:

$$
\left(\begin{array}{c}
\frac{\partial}{\partial x_{1}} \\
\frac{\partial}{\partial x_{2}} \\
\frac{\partial}{\partial x_{3}}
\end{array}\right) U_{l}={ }^{t} J_{0}\left(\begin{array}{c}
\frac{\partial}{\partial \phi_{1}} \\
\frac{\partial}{\partial \phi_{2}} \\
\frac{\partial}{\partial \phi_{3}}
\end{array}\right) \tilde{U}_{l}={ }^{t} J_{0}\left(\begin{array}{c}
\kappa_{l} \frac{1}{\tilde{\Gamma}} \tilde{U}_{l} \\
\frac{\partial \tilde{U}_{l}}{\partial \phi_{2}} \\
\frac{\partial \tilde{U}_{l}}{\partial \phi_{3}}
\end{array}\right)=\frac{\kappa_{1}^{\prime}}{\Gamma N_{0}}{ }^{t} J_{1}^{-1}\left(\begin{array}{l}
\frac{\kappa_{l}}{\kappa_{1}^{\prime}} \tilde{U}_{l} \\
\frac{\partial \tilde{U}_{l}}{\partial \phi_{2}} \\
\frac{\partial \tilde{U}_{l}}{\partial \phi_{3}}
\end{array}\right) .
$$

Note that, from the "dimensional" point of view, to derivate is equivalent to multiplying by $\left(\Gamma N_{0}\right)^{-1}$.

Remark. Transforms of second order derivatives. Now (92) is also written as

$$
\frac{\partial U_{l}}{\partial x_{j}}=\frac{\kappa_{1}^{\prime}}{\Gamma N_{0}} V_{l, j}, \quad \text { with } V_{l, j}=\frac{\kappa_{l}}{\kappa_{1}^{\prime}} \eta_{j} \tilde{U}_{l}+\delta_{j} \frac{\partial \tilde{U}_{l}}{\partial \phi_{2}}+\varepsilon_{j} \frac{\partial \tilde{U}_{l}}{\partial \phi_{3}} .
$$

Then the second order derivatives of $U_{l}$ are:

$$
\left\{\begin{aligned}
\frac{\partial}{\partial x_{k}} \frac{\partial U_{l}}{\partial x_{j}} & =\frac{\kappa_{1}^{\prime}}{\left(\Gamma N_{0}\right)^{2}}\left[-\eta_{k} \kappa_{1}^{\prime} V_{l, j}+\eta_{k} \kappa_{l} V_{l, j}+\kappa_{1}^{\prime}\left(\delta_{k} \frac{\partial V_{l, j}}{\partial \phi_{2}}+\varepsilon_{k} \frac{\partial V_{l, j}}{\partial \phi_{3}}\right)\right] \\
& =\frac{\kappa_{1}^{\prime}}{\left(\Gamma N_{0}\right)^{2}}\left[\left(\kappa_{l}-\kappa_{1}^{\prime}\right) \eta_{k} V_{l, j}+\kappa_{1}^{\prime}\left(\delta_{k} \frac{\partial V_{l, j}}{\partial \phi_{2}}+\varepsilon_{k} \frac{\partial V_{l, j}}{\partial \phi_{3}}\right)\right] .
\end{aligned}\right.
$$

This derivation is equivalent to a multiplication by $\left(\Gamma N_{0}\right)^{-2}$ (again from the "dimensional" point of view), and thus the used transformation does not allows us to treat equations with second order terms (with the Laplacian for example) with constant coefficients. 


\section{Remarks.}

We recall that one of the main properties of the change of variables is to change the particle derivative into the partial derivative.

This method is a general method of variables separation in a system of equations with first order partial derivatives quasi-linear and non homogeneous.

In some sense the new variables are similar to Lagrangian coordinates, so that the MHD equations transformed by $J$ may be considered as an other version of the MHD equations in Lagrangian coordinates.

\subsection{Solution of the simplified MHD equations}

\subsubsection{Some remarks about the self similar solutions}

The dimensions of $(\rho, v, H, p)$ with respect to $\beta_{1}, \tilde{\beta}_{1}$ are, with $\theta_{1}^{\prime}=\theta-\theta_{0}, \kappa_{1}^{\prime}=\kappa-\kappa_{0}$

$$
\begin{aligned}
& \nu_{\beta_{1}}(\rho)=2 \theta_{0}, \quad \nu_{\beta_{1}}(v)=2 \theta_{1}^{\prime}, \quad \nu_{\beta_{1}}(H)=\theta, \quad \nu_{\beta_{1}}(p)=2 \theta, \\
& \nu_{\tilde{\beta}_{1}}(\rho)=2 \kappa_{0}, \quad \nu_{\tilde{\beta}_{1}}(v)=2 \kappa_{1}^{\prime}, \quad \nu_{\tilde{\beta}_{1}}(H)=\kappa, \quad \nu_{\tilde{\beta}_{1}}(p)=2 \kappa \text {. }
\end{aligned}
$$

This corresponds to

$$
\begin{cases}\tilde{\rho}=\beta_{1}^{2 \theta_{0}} \tilde{\beta}_{1}^{2 \kappa_{0}} \tilde{\tilde{\rho}}, & \tilde{v}=\beta_{1}^{\theta-\theta_{0}} \tilde{\beta}_{1}^{\kappa-\kappa_{0}} \tilde{\tilde{v}} \\ \tilde{H}=\beta_{1}^{\theta} \tilde{\beta}_{1}^{\kappa} \tilde{\tilde{H}}, & \tilde{p}=\beta_{1}^{2 \theta} \tilde{\beta}_{1}^{2 \kappa} \tilde{\tilde{p}}\end{cases}
$$

with

$$
\tilde{\tilde{\rho}}=W_{1}, \quad \tilde{\tilde{v}}_{j}=W_{1+j}, \quad \tilde{\tilde{H}}_{j}=W_{4+j}, \quad j=1,2,3, \quad \tilde{\tilde{p}}=W_{8} .
$$

Let us calculate $\theta_{0}, \theta$. First note the very simple value of $\operatorname{div} v[\mathrm{using}(63)]$ :

$$
\operatorname{div} v=\frac{\alpha_{1}}{\Gamma} \operatorname{div} x=3 \frac{\alpha_{1}}{\Gamma} .
$$

From the conservative mass (Eq. (15i)), we get: $\theta+2 \alpha_{1}=0$. From the adiabatic evolution (Eq. (17')): $\theta-\gamma \theta_{0}=0$. From the evolution equation of $H$ (if $H$ is not identically 0 ): $2 \theta_{0}+3 \alpha_{1}=0$.

Thus, we obtain $\gamma, \theta$ and $\theta_{0}$ as

$$
\gamma=\frac{4}{3}, \quad \theta_{0}=-\frac{3}{2} \alpha_{1}, \quad \theta=-2 \alpha_{1} .
$$

The metric separation equation gives the functions $\beta_{1}$ and $M$

$$
\beta_{1}(\tau)=C_{1}\left(\frac{\tau+\tau_{0}}{\tau_{0}}\right)^{2 /\left(3 \alpha_{1}\right)}, \quad M(\tau)=\left(\frac{\tau+\tau_{0}}{\tau_{0}}\right)^{2 / 3} .
$$

The solution must satisfy the Euler equation and $\operatorname{div} H=0$. Using (96) in the Euler equation, we verify that we obtain an equation with the only variables $\phi_{2}$ and $\phi_{3}$ (according to the method of separations of variables). For the equation $\operatorname{div} H=0$, we obtain:

$$
\frac{\kappa}{\kappa_{1}^{\prime}} \eta \cdot \tilde{\tilde{H}}+\left(\delta \cdot \frac{\partial \tilde{\tilde{H}}}{\partial \phi_{2}}+\varepsilon \cdot \frac{\partial \tilde{\tilde{H}}}{\partial \phi_{3}}\right)=0 .
$$




\subsubsection{Solution of the whole MHD system}

We can entirely solve the system using spherical coordinates on the unit sphere $\Sigma$, with the usual notations $\phi_{2}=\theta, \phi_{3}=\phi$

$\underline{\text { Use of the matrix } J_{1} \text { and its inverse } J_{1}^{-1} \text {. Solution of } \operatorname{div} H=0}$

The Jacobian matrix is [see (87)]:

$$
J_{1}=\left(w, \frac{\partial w}{\partial \theta}, \frac{\partial w}{\partial \phi}\right)=|w|\left(\alpha, \frac{\partial \alpha}{\partial \theta}, \frac{\partial \alpha}{\partial \phi}\right)
$$

with $\alpha=e_{r}=(\sin \theta \cos \phi, \sin \theta \sin \phi, \cos \theta)$. Thus the transposed matrix of the inverse of $J_{1}$ is [see $(89)$ ]

$$
{ }^{t} J_{1}^{-1}=(\eta, \delta, \varepsilon)=\frac{1}{|w|}\left(e_{r}, e_{\theta}, \frac{1}{\sin \theta} e_{\phi}\right)
$$

Therefore, with $H=H_{r} e_{r}+H_{\Sigma}=H_{r} e_{r}+H_{\theta} e_{\theta}+H_{\phi} e_{\phi}$ we have

$$
H \cdot \eta|w|=H_{r}, \quad H \cdot \delta|w|=H_{\theta}, \quad H \cdot \varepsilon|w|=\frac{1}{\sin \theta} H_{\phi}
$$

Then the equation $\operatorname{div} H=0,(100)$, gives with the spherical surface divergence $\operatorname{div}_{\Sigma}$

$$
\nu_{0} H_{r}+\operatorname{div}_{\Sigma} H_{\Sigma}=0, \quad \text { with } \nu_{0}=\frac{\kappa}{\kappa_{1}^{\prime}}+2
$$

We obtain (104) also by using the div in spherical coordinates

$$
\frac{\partial H_{r}}{\partial r}+\frac{2}{r} H_{r}+\operatorname{div}_{\Sigma} H_{\Sigma}=0
$$

and the transformation due to (50)

$$
J\left(r \frac{\partial}{\partial r}\right)=J\left(\sum x_{i} \frac{\partial}{\partial x_{i}}\right)=\frac{\Gamma}{\alpha_{1}} J\left(\sum v_{i} \frac{\partial}{\partial x_{i}}\right)=-\frac{\Gamma}{\alpha_{1}} \frac{\partial \phi_{1}}{\partial t^{\prime}} \frac{\partial}{\partial \phi_{1}}=\frac{\Gamma}{\alpha_{1}} \frac{\alpha_{1}}{\kappa_{1}^{\prime}} \frac{1}{G} \frac{\partial}{\partial \phi_{1}}=\frac{\tilde{\Gamma}}{\kappa_{1}^{\prime}} \frac{\partial}{\partial \phi}
$$

and with $J\left(r \frac{\partial}{\partial r}\right) \tilde{H}=\frac{\kappa}{\kappa_{1}^{\prime}} \tilde{H}$.

$\underline{\text { Solution of Euler equation }}$

We take

$$
\rho \frac{\mathrm{d} v}{\mathrm{~d} t}+\operatorname{grad} p=F_{L}=\mu_{0} \operatorname{rot} H \times H
$$

and we project on the plane tangent to the unit sphere. The equation

$$
\operatorname{grad}_{\Sigma} p=\Pi_{\Sigma} F_{L}=\mu_{0} \Pi_{\Sigma}(\operatorname{rot} H \times H)
$$

gives $p$ through $H$ up to a constant. We get $\rho$ through $H$ and $p$ by a projection of the Euler equation on the radial vector. 
Then we take the surface curl, i.e., $\left(\operatorname{rot}_{\Sigma}\right)$ of this equation and we obtain the equation on $H$ only

$$
\operatorname{rot}_{\Sigma} \Pi_{\Sigma}(\operatorname{rot} H \times H)=0 .
$$

We couple this equation with $\operatorname{div} H=0$ in order to obtain $H$.

$\underline{\text { Solution of the system of equations }}$

We can write equation (108) in the form:

$$
\operatorname{rot}_{\Sigma} \Pi_{\Sigma}(\operatorname{rot} H \times H)=\alpha \cdot \operatorname{rot}(\operatorname{rot} H \times H), \quad \text { with } \alpha=e_{r} .
$$

Using differential geometry (see $[5,6]$ ), we obtain:

$$
H_{\Sigma} \cdot\left(\operatorname{grad}_{\Sigma} \operatorname{rot}_{\Sigma} H_{\Sigma}+\lambda \overrightarrow{\operatorname{rot}}_{\Sigma} \operatorname{div}_{\Sigma} H_{\Sigma}\right)=0, \quad \text { with } \lambda=-\frac{\kappa+\kappa_{1}^{\prime}}{\kappa+2 \kappa_{1}^{\prime}},
$$

or also:

$$
\left(e_{r} \times H_{\Sigma}\right) \cdot\left(\overrightarrow{\operatorname{rot}}_{\Sigma} \operatorname{rot}_{\Sigma} H_{\Sigma}-\lambda \operatorname{grad}_{\Sigma} \operatorname{div}_{\Sigma} H_{\Sigma}\right)=0 .
$$

Instead of the vector unknown $H_{\Sigma}$, let $f_{1}, f_{2}$ be two scalar unknowns with

$$
\operatorname{div}_{\Sigma} H_{\Sigma}=f_{1}, \quad \operatorname{rot}_{\Sigma} H_{\Sigma}=f_{2}, \quad \text { and } f_{1}=-\left(2+\frac{\kappa}{\kappa_{1}^{\prime}}\right) H_{r}, \quad(\operatorname{div} H=0) .
$$

Now, we can get $H_{\Sigma}$ through $f_{1}, f_{2}$. Using the Hodge decomposition (see [5]) of $H_{\Sigma}$ into:

$$
H_{\Sigma}=\operatorname{grad}_{\Sigma} \zeta_{1}+\overrightarrow{\operatorname{rot}}_{\Sigma} \zeta_{2}, \quad \text { with } \zeta_{i} \in H^{1}(\Sigma), \quad i=1,2
$$

$\left(H^{1}(\Sigma)\right.$ is the Sobolev space on the sphere) we obtain: $f_{i}=\Delta_{\Sigma} \zeta_{i}, i=1,2$, thus:

$$
H_{\Sigma}=\operatorname{grad}_{\Sigma} G f_{1}+\overrightarrow{\operatorname{rot}}_{\Sigma} G f_{2}, \quad \text { and } e_{r} \times H_{\Sigma}=\overrightarrow{\operatorname{rot}}_{\Sigma} G f_{1}-\operatorname{grad}_{\Sigma} G f_{2}
$$

where $G$ is the inverse of the Laplace-Beltrami operator on the sphere in the space $L_{0}^{2}(\Sigma)$ (orthogonal to the constant functions), given by

$$
G f(\alpha)=\int_{\Sigma} g(\alpha, \beta) f(\beta) \mathrm{d} \Sigma_{\beta} \quad \text { with } g(\alpha, \beta)=\frac{1}{4 \pi} \log \frac{1-\alpha \cdot \beta}{2} .
$$

Equation (110) is thus reduced to:

$$
\begin{aligned}
\left(\overrightarrow{\operatorname{rot}}_{\Sigma} G f_{1}-\operatorname{grad}_{\Sigma} G f_{2}\right) \cdot\left(-\lambda \operatorname{grad}_{\Sigma} f_{1}+\overrightarrow{\operatorname{rot}}_{\Sigma} f_{2}\right) & =0, \quad \text { i.e., } \\
\left(\overrightarrow{\operatorname{rot}}_{\Sigma} G f_{1}-\operatorname{grad}_{\Sigma} G f_{2}\right) \cdot e_{r} \times\left(\lambda \overrightarrow{\operatorname{rot}}_{\Sigma} f_{1}+\operatorname{grad}_{\Sigma} f_{2}\right) & =0 .
\end{aligned}
$$

Then, from the magnetic field $H$, we get the other unknowns, the pressure $p$ and the density $\rho$. We get $p$ through (107):

$$
\frac{1}{\mu_{0}} \operatorname{grad}_{\Sigma} p=\Pi_{\Sigma}(\operatorname{rot} H \times H)=H_{r}\left(\left(1+\frac{\kappa}{\kappa_{1}^{\prime}}\right) H_{\Sigma}-\operatorname{grad}_{\Sigma} H_{r}\right)+\left(\operatorname{rot}_{\Sigma} H_{\Sigma}\right) e_{r} \times H_{\Sigma},
$$


and then $\rho$ through the projection of (106) on the unit radial vector:

$$
\rho \frac{\mathrm{d} v_{r}}{\mathrm{~d} t}+\frac{\partial p}{\partial r}=\mu_{0} \alpha \cdot F, \quad \text { with } \alpha \cdot F=\alpha \cdot(\operatorname{rot} H \times H) .
$$

Using (105), we have

$$
\frac{\mathrm{d} v_{r}}{\mathrm{~d} t}=\frac{\theta_{1}^{\prime}}{\Gamma} v_{r}, \quad v_{r}=\frac{\alpha_{1}}{\Gamma} r \quad \text { thus } \quad \frac{\mathrm{d} v_{r}}{\mathrm{~d} t}=\frac{\theta_{1}^{\prime}}{\alpha_{1}} \frac{v_{r}^{2}}{r} \quad \text { and } J\left(\frac{\partial}{\partial r}\right) \tilde{p}=\frac{2 \kappa}{\kappa_{1}^{\prime}} \cdot \frac{1}{r} \tilde{p}
$$

Therefore (118) gives

$$
\lambda_{0} \rho \frac{|v|^{2}}{2}+p=\lambda_{1} r \alpha \cdot F=\lambda_{1} x \cdot F, \quad \text { with } \quad \lambda_{0}=\frac{\theta_{1}^{\prime}}{\alpha_{1}} \frac{\kappa_{1}^{\prime}}{\kappa}, \quad \lambda_{1}=\frac{\mu_{0}}{2} \frac{\kappa_{1}^{\prime}}{\kappa}
$$

We also have with (104) and $\lambda$ given by (110):

$$
\begin{aligned}
\alpha \cdot F & =-\left(e_{r} \times H\right) \cdot(\operatorname{rot} H)_{\Sigma}=-\left(e_{r} \times H\right) \cdot\left(\left(1+\frac{\kappa}{\kappa_{1}^{\prime}}\right) e_{r} \times H-\overrightarrow{\operatorname{rot}}_{\Sigma} H_{r}\right) \\
& =-\left(1+\frac{\kappa}{\kappa_{1}^{\prime}}\right)\left|H_{\Sigma}\right|^{2}+H_{\Sigma} \cdot \operatorname{grad}_{\Sigma} H_{r}=-\left(2+\frac{\kappa}{\kappa_{1}^{\prime}}\right) H_{\Sigma} \cdot\left(\lambda H_{\Sigma}+\operatorname{grad}_{\Sigma} \operatorname{div}_{\Sigma} H_{\Sigma}\right) .
\end{aligned}
$$

We get particular solutions of (116) with a given polarization using (real) spherical harmonics. Let $K_{n}$ be a real spherical harmonic of order $n(n$ integer, $n \neq 0)$

$$
\Delta_{\Sigma} K_{n}=-n(n+1) K_{n}
$$

then we have:

TE polarization, thus $\left(f_{1}, f_{2}\right)=\left(K_{n}, 0\right)$

$$
H_{r}=-\frac{1}{\nu_{0}} K_{n}, \quad H_{\Sigma}=-\frac{1}{n(n+1)} \operatorname{grad}_{\Sigma} K_{n} \quad \text { on } \Sigma
$$

TM polarization, thus $\left(f_{1}, f_{2}\right)=\left(0, K_{n}\right)$

$$
H_{\Sigma}=-\frac{1}{n(n+1)} \overrightarrow{\operatorname{rot}}_{\Sigma} K_{n}, \quad H_{r}=0 .
$$

(i) In the case of TE polarization, we get:

$$
\begin{aligned}
\Pi_{\Sigma}(\operatorname{rot} H \times H) & =\left(\nu_{0}-1\right) H_{r} H_{\Sigma}-H_{r} \operatorname{grad}_{\Sigma} H_{r}=\frac{\tilde{\lambda}_{n}}{2} \operatorname{grad}_{\Sigma}\left|K_{n}\right|^{2} \\
\text { with } \tilde{\lambda}_{n} & =\frac{1}{\nu_{0}^{2}}\left(\frac{\nu_{0}\left(\nu_{0}-1\right)}{n(n+1)}-1\right), \quad \nu_{0} \text { given by }(104)
\end{aligned}
$$

Thus from (117)

$$
\operatorname{grad}_{\Sigma} p=\mu_{0} \frac{\tilde{\lambda}_{n}}{2} \operatorname{grad}_{\Sigma}\left(\left|K_{n}\right|^{2}\right)
$$


therefore

$$
\left.p\right|_{\Sigma}=\mu_{0} \frac{\tilde{\lambda}_{n}}{2}\left|K_{n}\right|^{2}=\mu_{0} \frac{\tilde{\lambda}_{n}}{2}\left|\nu_{0}\right|^{2}\left|H_{r}\right|^{2}, \quad \text { with } \nu_{0}=2+\frac{\kappa}{\kappa_{1}^{\prime}} .
$$

We also have

$$
\alpha \cdot F=-\left(\nu_{0}-1\right)\left|H_{\Sigma}\right|^{2}+H_{\Sigma} \cdot \operatorname{grad}_{\Sigma} H_{r}=\nu_{0}\left[\frac{\nu_{0}-1}{\nu_{0}}+n(n+1)\right]\left|H_{\Sigma}\right|^{2}
$$

thus, with (122):

$$
\alpha \cdot F=\nu_{0}\left[\frac{\nu_{0}-1}{\nu_{0}}+n(n+1)\right] \frac{1}{n(n+1)}\left|K_{n}\right|^{2}
$$

Therefore (120) is

$$
\rho \frac{v^{2}}{2}=\frac{\lambda_{1}}{\lambda_{0}} \alpha \cdot F-\frac{1}{\lambda_{0}} p=-\mu_{0} \alpha \cdot F+2\left(\nu_{0}-2\right) p=\mu_{0}\left[-2 \frac{1}{n(n+1)} \frac{\left(\nu_{0}-1\right)^{2}}{\nu_{0}}-\nu_{0}+\frac{\nu_{0}-2}{\nu_{0}^{2}}\right]\left|K_{n}\right|^{2} .
$$

The function $p$ is positive if and only if $\tilde{\lambda}_{n} \geq 0$, i.e., $n(n+1) \leq \nu_{0}\left(\nu_{0}-1\right)$ that is $n-1 \leq \nu_{0}$ or also $-n-2 \geq \nu_{0}$ [with $\nu_{0}$ given by (104)].

Then from (128), we see that when $-n-2 \geq \nu_{0}, \rho$ is positive; but $\rho$ is negative if $\nu_{0}$ is positive; thus we obtain that when $\nu_{0}$ is negative, with $-n-2 \geq \nu_{0}, n$ a (non zero) positive integer, $p$ and $\rho$ are positive as wanted for pressure and specific mass. Moreover, with given negative $\nu_{0}$, we have an upper bound for $n$ : only a finite number of spherical Harmonics are acceptable in TE-polarization.

(ii) In the case of TM polarization, we get:

$$
\left.p\right|_{\Sigma}=\mu_{0} \frac{1}{n(n+1)} \frac{\left|K_{n}\right|^{2}}{2}
$$

and

$$
\alpha \cdot F=-\left(\nu_{0}-1\right)\left|H_{\Sigma}\right|^{2}=-\frac{\left(\nu_{0}-1\right)}{[n(n+1)]^{2}}\left|\overrightarrow{\operatorname{rot}}_{\Sigma} K_{n}\right|^{2}=-\frac{\left(\nu_{0}-1\right)}{n(n+1)}\left|K_{n}\right|^{2} .
$$

Thus

$$
\rho \frac{v^{2}}{2}=-\mu_{0} \alpha \cdot F+2\left(\nu_{0}-2\right) p=\mu_{0} \frac{1}{n(n+1)}\left(2 \nu_{0}-3\right)\left|K_{n}\right|^{2}
$$

We see that $p$ is always positive; $\rho$ is positive if $\nu_{0}>3 / 2$. Thus when $\nu_{0}>3 / 2$, we obtain that $p$ and $\rho$ are positive as wanted for pressure and specific mass. There is no bound on $n$ in TM-polarization: all spherical Harmonics with $n \neq 0$ are acceptable in TM-polarization.

Regularity of the magnetic field at 0 .

Note that the behavior of $H$ in a neighborhood of 0 is of type $r^{\kappa / \kappa_{1}^{\prime}}$, thus $H$ is finite at 0 if $\kappa / \kappa_{1}^{\prime} \geq 0$, thus if $\nu_{0} \geq 2$. Using the formula

$$
H_{j}=\beta_{1}^{\theta} \tilde{\beta}_{1}^{\kappa} W_{4+j}, \quad \text { with } \beta_{1}=C_{1}\left(\frac{\tau+\tau_{0}}{\tau_{0}}\right)^{2 / 3 \alpha_{1}}, \quad \tilde{\beta}_{1}^{\kappa}=C \beta_{1}^{-\alpha_{1} \kappa / \kappa_{1}^{\prime}} r^{\kappa / \kappa_{1}^{\prime}}
$$


we have

$$
H_{j}=\beta_{1}^{-\alpha_{1} \nu_{0}} r^{\kappa / \kappa_{1}^{\prime}} W_{4+j}=C_{1}^{-\alpha_{1} \nu_{0}}\left(\frac{\tau+\tau_{0}}{\tau_{0}}\right)^{-\frac{2}{3} \nu_{0}} r^{\kappa / \kappa_{1}^{\prime}} W_{4+j}
$$

In TM-polarization, the magnetic field is regular at 0 if $\kappa / \kappa_{1}^{\prime} \geq 0$, i.e., if $\nu_{0} \geq 2$.

In the case of $T E$ polarization, the magnetic field $H$ is:

$$
H_{\Sigma}=-\frac{1}{n(n+1)} \beta_{1}^{-\alpha_{1} \nu_{0}} r^{\kappa / \kappa_{1}^{\prime}} \operatorname{grad}_{\Sigma} K_{n}, \quad H_{r}=-\frac{1}{\nu_{0}} \beta_{1}^{-\alpha_{1} \nu_{0}} r^{\kappa / \kappa_{1}^{\prime}} K_{n} .
$$

Thus (for example) the radial component is

$$
H_{r}(x, t)=-\frac{1}{\nu_{0}} C_{1}^{-\alpha_{1} \nu_{0}}\left(\frac{\tau+\tau_{0}}{\tau_{0}}\right)^{-\frac{2}{3} \nu_{0}} r^{\kappa / \kappa_{1}^{\prime}} K_{n}, \quad r=|x| .
$$

We see that when $\nu_{0}$ is negative the magnetic field in TE-polarization is singular at 0 .

\subsubsection{Conclusion}

We summarize the particular solution obtained with a variable separation method for this simplified MHD problem. We write the unknowns, the data and the constants.

Let $\hat{\beta}_{1}$ be

$$
\beta_{1}(\tau)=C_{1} \hat{\beta}_{1}(\tau), \quad \text { with } C_{1}=\beta_{1}(0), \quad \text { and } \hat{\beta}_{1}(0)=1
$$

Then (45) [or $\left.\left(96^{\prime}\right)\right]$ is

$$
\tilde{U}_{m}(\tau, \phi)=\hat{\beta}_{1}^{\theta_{m}}(\tau) \tilde{\beta}_{1}^{\kappa_{m}}\left(\phi_{1}\right) \tilde{W}_{m}\left(\phi_{2}, \phi_{3}\right), \quad \text { with } \quad \tilde{W}_{m}\left(\phi_{2}, \phi_{3}\right)=C_{1}^{\theta_{m}} W_{m}\left(\phi_{2}, \phi_{3}\right),
$$

and $\tilde{\beta}_{1}$ is such that

$$
\tilde{\beta}_{1}^{\kappa_{1}^{\prime}}\left(\phi_{1}\left(x_{a}(t), t\right)\right)=\mu|a|, \quad \text { with } \mu=\tilde{C} \beta_{1}^{-\alpha_{1}}(0)=\tilde{C} C_{1}^{-\alpha_{1}}
$$

[see $(65,66)]$. Thus $(66)$ is: $\tilde{\beta}_{1}^{\kappa_{1}^{\prime}}\left(\phi_{1}\left(x, t^{\prime}\right)\right)=\mu \hat{\beta}_{1}^{-\alpha_{1}}\left(t^{\prime}\right) r$.

If we take $|w|=1$, then $\tilde{C} \cdot \Gamma(0) \beta_{1}^{\theta_{1}^{\prime}-\alpha_{1}} / \kappa_{1}^{\prime}=1$ [see (67)]. If we take $w$ dimensionless, the dimensions of the constants $\tilde{C}, \mu$ are

$$
\nu(\tilde{C})=\left(\alpha_{1}-\theta_{1}^{\prime}\right) \nu\left(\beta_{1}\right)+(0,-1,0), \quad \nu(\mu)=\kappa_{1}^{\prime} \nu\left(\tilde{\beta}_{1}\right)+(-1,0,0) \quad \text { with } \nu(\Gamma)=(0,1,0) .
$$

Then the unknowns are given by:

$$
\begin{cases}\rho\left(x_{a}(t), t\right)=C_{\rho}|a|^{2 \kappa_{0} / \kappa_{1}^{\prime}} \hat{\beta}_{1}^{2 \theta_{0}}(t) \tilde{\tilde{\rho}}(\alpha), & v\left(x_{a}(t), t\right)=C_{v}|a| \hat{\beta}_{1}^{\theta_{1}^{\prime}}(t) \tilde{\tilde{v}}(\alpha), \\ H\left(x_{a}(t), t\right)=C_{H}|a|^{\kappa / \kappa_{1}^{\prime}} \hat{\beta}_{1}^{\theta}(t) \tilde{\tilde{H}}(\alpha), & p\left(x_{a}(t), t\right)=C_{p}|a|^{2 \kappa / \kappa_{1}^{\prime}} \hat{\beta}_{1}^{2 \theta}(t) \tilde{\tilde{p}}(\alpha),\end{cases}
$$

with $\alpha=a /|a|$, and with the constants:

$$
\left\{\begin{array}{ll}
C_{\rho}=C_{1}^{2 \theta_{0}} \mu^{2 \kappa_{0} / \kappa_{1}^{\prime}}, & C_{v}=C_{1}^{\theta_{1}^{\prime}} \mu \\
C_{H}=C_{1}^{\theta} \mu^{\kappa / \kappa_{1}^{\prime}}, & C_{p}=C_{1}^{2 \theta_{0}} \mu^{2 \kappa / \kappa_{1}^{\prime}} .
\end{array} \quad\left(\text { thus } C_{v}=C_{1}^{\theta_{1}^{\prime}-\alpha_{1}} \tilde{C}=\frac{\kappa_{1}^{\prime}}{\Gamma(0)}\right),\right.
$$


Then the unknowns are obtained through initial conditions $(t=0)$ on the unit sphere $\Sigma(|a|=1)$

$$
\begin{cases}\rho_{0}(\alpha)=C_{\rho} \tilde{\tilde{\rho}}(\alpha), & v_{0}(\alpha)=C_{v} \tilde{\tilde{v}}(\alpha)=\frac{\alpha_{1}}{\Gamma(0)} \alpha, \\ H_{0}(\alpha)=C_{H} \tilde{\tilde{H}}(\alpha), & p_{0}(\alpha)=C_{p} \tilde{\tilde{p}}(\alpha), \quad \alpha \in \Sigma=S^{2} .\end{cases}
$$

These quantities must satisfy very simple equations, and we have given explicit formulas in Section 3.4.2.

The method with separate variables applied to the MHD problem allows us to exhibit a family of explicit self similar solutions.

These solutions are radial with respect to the motion of the fluid particle, but are not invariant with respect to rotations! We have exhibited completely explicit solutions with spherical harmonics for the magnetic field, corresponding to particular polarizations (TE or TM) of the field.

The case without magnetic field. Self similar solutions of the compressible fluid equations

Let $H=0$. Only the Euler equation remains:

$$
\rho \frac{\mathrm{d} v}{\mathrm{~d} t}+\operatorname{grad} p=0
$$

The conservative law of mass gives

$$
2 \theta_{0}+3 \alpha_{1}=0
$$

and the relation $p \rho^{-\gamma}$ constant (for any given $\gamma>1$ ) leads to

$$
\theta-\gamma \theta_{0}=0
$$

We project the Euler equation on $v$ and we obtain:

$$
\frac{1}{2} \rho v^{2}+\lambda p=0, \quad \text { with } \lambda=\frac{\kappa}{\kappa_{1}^{\prime}} \frac{\alpha_{1}}{\theta-\theta_{0}}=-\frac{2}{3(\gamma-1)} \frac{\kappa}{\kappa_{1}^{\prime}}
$$

(thus $\lambda<0$, so that $\kappa$ and $\kappa_{1}^{\prime}$ must have the same sign). From the relation

$$
\frac{\mathrm{d} v}{\mathrm{~d} t}=\frac{\theta_{1}^{\prime}}{\Gamma} v=\frac{\theta_{1}^{\prime} \alpha_{1}}{\Gamma^{2}} x_{a}
$$

$\mathrm{d} v / \mathrm{d} t$ is along the radial vector, and so is $\operatorname{grad} p$. Therefore $\operatorname{grad}_{\Sigma} p=0$ and $p$ is a constant quantity on the sphere. Consequently, with the notations (139), $\tilde{\tilde{p}}(\alpha)$ is a constant, independent of $\alpha$. By the formula (145), so is $\tilde{\tilde{p}}(\alpha)$.

Thus, our particular (self similar) solutions of the compressible fluid equations are:

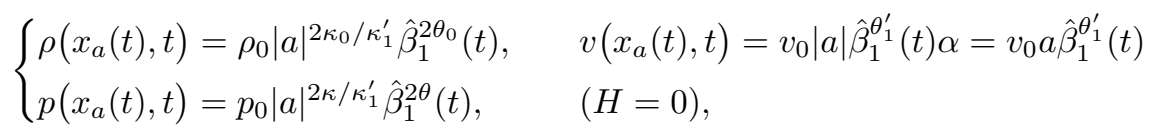

with positive constants $\left(\rho_{0}, v_{0}, p_{0}\right)$ satisfying

$$
\frac{1}{2} \rho_{0} v_{0}^{2}+\lambda p_{0}=0
$$


The exponents of $\hat{\beta}_{1}(t)$ are such that

$$
2 \theta_{0}+3 \alpha_{1}=0, \quad \theta-\gamma \theta_{0}=0, \quad \theta_{1}^{\prime}=\theta-\theta_{0}=(\gamma-1) \theta_{0}, \quad \text { and } \alpha_{1}-\theta_{1}^{\prime}=-\left(\gamma-\frac{1}{3}\right) \theta_{0} \neq 0
$$

Now $\hat{\beta}_{1}$ is given by $\hat{\beta}_{1}=\left(\frac{t+\tau_{0}}{\tau_{0}}\right)^{\lambda_{1}}$ with $\tau_{0}$ a positive constant, and

$$
\lambda_{1}=\left(\alpha_{1}-\theta_{1}^{\prime}\right)^{-1}=-\theta_{0}^{-1}\left(\gamma-\frac{1}{3}\right)^{-1}
$$

Therefore, the time evolutions of $(\rho, p, v)$ are respectively given through the terms:

$$
\hat{\beta}_{1}^{2 \theta_{0}}(t)=\left(\frac{t+\tau_{0}}{\tau_{0}}\right)^{-\frac{2}{\gamma-1 / 3}}, \quad \hat{\beta}_{1}^{2 \theta}(t)=\left(\frac{t+\tau_{0}}{\tau_{0}}\right)^{-\frac{2 \gamma}{\gamma-1 / 3}}, \quad \hat{\beta}_{1}^{\theta_{1}^{\prime}}(t)=\left(\frac{t+\tau_{0}}{\tau_{0}}\right)^{-\frac{\gamma-1}{\gamma-1 / 3}} .
$$

This proves that all the quantities $\rho,|v|, p$ are decreasing functions of time. $\left(\right.$ But $M(t)=\hat{\beta}_{1}^{\alpha_{1}}(t)=\left(\frac{t+\tau_{0}}{\tau_{0}}\right)^{\lambda_{1} \alpha_{1}}=$ $\left(\frac{t+\tau_{0}}{\tau_{0}}\right)^{\frac{2}{3 \gamma-1}}$ is an increasing function).

Also, we have: $\kappa_{1}^{\prime}=\kappa-\kappa_{0}$ and $\lambda_{1}$ must be negative, so that $\kappa$ and $\kappa_{1}^{\prime}$ have the same sign. The exponent $2 \kappa / \kappa_{1}^{\prime}$ is positive, thus $p$ is an increasing function of $|a|$.

Let $x=x_{a}(t)=M(t) a$. Now with usual Euler coordinates, we can give relations (147) in a simpler and "classical" form (similar to "usual" self similar solution, see for example [11]): the velocity is $v(x, t)=v_{0} \frac{t_{0}}{t+t_{0}} x$, thus the radial component is $v_{r}=v_{0} \frac{t_{0}}{t+t_{0}} r, r=|x|$ and we have:

$$
\rho(x, t)=\rho_{0}\left(\frac{t_{0}}{t+t_{0}}\right)^{s} \frac{1}{r^{k+3}}, \quad p(x, t)=p_{0}\left(\frac{t_{0}}{t+t_{0}}\right)^{s+2} \frac{1}{r^{k+1}},
$$

with new constants $k, s, k+1=-2 \kappa / \kappa_{1}^{\prime}=-2 \nu_{0}+4, s=\frac{2}{3 \gamma-1}\left(3+2\left(\kappa_{0} / \kappa_{1}^{\prime}\right)\right)$ thus we have:

$$
k=-2 \nu_{0}-3, \quad s=-\frac{2 k}{3 \gamma-1}
$$

Recall also (148) with $\lambda$ given by (145), that is $\lambda=(k+1) / 3(\gamma-1)$.

We emphasize that we did not assume a priori that the evolution was radial! Again the solution is non singular at 0 .

The case with magnetic field. Self similar solutions of the simplified MHD equations

Our self similar solution of the MHD equations is given by (139), with

$$
\left\{\begin{array}{ll}
\hat{\beta}_{1}^{2 \theta_{0}}(t)=\left(\frac{t+\tau_{0}}{\tau_{0}}\right)^{-2}, & \hat{\beta}_{1}^{2 \theta}(t)=\left(\frac{t+\tau_{0}}{\tau_{0}}\right)^{-\frac{8}{3}}, \\
\hat{\beta}_{1}^{\theta_{1}^{\prime}}(t)=\left(\frac{t+\tau_{0}}{\tau_{0}}\right)^{-\frac{1}{3}}, & \hat{\beta}_{1}^{\theta}(t)=\left(\frac{t+\tau_{0}}{\tau_{0}}\right)^{-\frac{4}{3}},
\end{array} \quad \text { with } M(t)=\left(\frac{t+\tau_{0}}{\tau_{0}}\right)^{\frac{2}{3}} .\right.
$$

All quantities $\rho,|v|,|H|, p)$ are decreasing functions of time, except for $M(t)$.

(a) Let $U^{0}(a)=\left.U(a, t)\right|_{t=0}, U^{0}(a)=\left(\rho^{0}(a), v^{0}(a), H^{0}(a), p^{0}(a)\right)$. 
These are the initial conditions. Then $U(t)$ can be written:

$$
U(t)=Z(t) U^{0}, \quad t \geq 0
$$

with

$$
Z(t)=\left(\hat{\beta}_{1}^{2 \theta_{0}}, I \hat{\beta}_{1}^{\theta_{1}^{\prime}}, I \hat{\beta}_{1}^{\theta}, \hat{\beta}_{1}^{2 \theta}\right)=\left(\left(\frac{t+\tau_{0}}{\tau_{0}}\right)^{-2}, I\left(\frac{t+\tau_{0}}{\tau_{0}}\right)^{-\frac{1}{3}}, I\left(\frac{t+\tau_{0}}{\tau_{0}}\right)^{-\frac{4}{3}},\left(\frac{t+\tau_{0}}{\tau_{0}}\right)^{-\frac{8}{3}}\right)
$$

with the identity matrix $I$. Note that $\left.Z(t)\right|_{t>0}$ is not a semi-group since

$$
Z\left(t_{1}\right) \cdot Z\left(t_{2}\right) \neq Z\left(t_{1}+t_{2}\right), \quad Z\left(t_{1}\right) \cdot Z\left(t_{2}\right) \neq Z\left(t_{1} \cdot t_{2}\right) .
$$

(b) Let (using polar coordinates):

$$
U^{0}(a)=U^{0}(|a|, \alpha), \quad \alpha=\frac{a}{|a|} \in \Sigma=S^{2}
$$

and let (as initial conditions on the unit sphere):

$$
\tilde{U}^{0}(\alpha)=U^{0}(1, \alpha)=\left.U^{0}(a)\right|_{a \in \Sigma} .
$$

Then the initial conditions $U^{0}(a)$ can be expressed with $\tilde{U}^{0}(\alpha)$ and $\Lambda(|a|)$ :

$$
\begin{aligned}
& \tilde{U}^{0}(a)=U^{0}(|a|, \alpha)=\Lambda(|a|) \tilde{U}^{0}(\alpha) \\
& \Lambda(|a|)=\left(|a|^{2 \kappa_{0} / \kappa_{1}^{\prime}}, I|a|, I|a|^{\kappa / \kappa_{1}^{\prime}},|a|^{2 \kappa / \kappa_{1}^{\prime}}\right),
\end{aligned}
$$

with $\kappa_{0} / \kappa_{1}^{\prime}=\left(\kappa-\kappa^{\prime}\right) / \kappa_{1}^{\prime}=\kappa / \kappa_{1}^{\prime}-1$ so that taking $\nu_{0}=\kappa / \kappa_{1}^{\prime}+2$, and $\nu_{0}>2$ we have

$$
\Lambda(|a|)=\left(|a|^{2 \kappa_{0} / \kappa_{1}^{\prime}}, I|a|, I|a|^{\kappa / \kappa_{1}^{\prime}},|a|^{2 \kappa / \kappa_{1}^{\prime}}\right)
$$

Note that $\Lambda(|a|)$ is a multiplicative group, which is a representation of the multiplicative group $\mathbb{R}^{+}$

$$
\Lambda\left(\left|a_{1}\right| \cdot\left|a_{2}\right|\right)=\Lambda\left(\left|a_{1}\right|\right) \cdot \Lambda\left(\left|a_{2}\right|\right)
$$

(c) Finally $U^{0}(a)$ is obtained by solving the equations $(107,111,117)$, so that our solution of the MHD equations is given by:

$$
U(t,|a|, \alpha)=Z(t) \Lambda(|a|) \tilde{U}^{0}(\alpha)
$$

Note that this solution is regular with time, for all $t>0$, and even for $t>-\tau_{0}$, with a singularity at $t=-\tau_{0}$. We emphasize that the solution is not singular at $t=0$, contrarily to usual self similar solutions.

Now using the Euler variable $x$ for $x_{a}(t)$ we obtain a simpler form to (139) [like (147')], the velocity is $v(x, t)=v_{0} \frac{t_{0}}{t+t_{0}} x$, thus the radial component is $v_{r}=v_{0} \frac{t_{0}}{t+t_{0}} r, r=|x|$ and we have:

$$
\begin{cases}\rho(x, t)=\rho_{0}(\alpha)\left(\frac{t_{0}}{t+t_{0}}\right)^{s} \frac{1}{r^{k+3}}, & p(x, t)=p_{0}(\alpha)\left(\frac{t_{0}}{t+t_{0}}\right)^{s+2} \frac{1}{r^{k+1}}, \\ H(x, t)=H_{0}(\alpha)\left(\frac{t_{0}}{t+t_{0}}\right)^{(s+2) / 2} \frac{1}{r^{(k+1) / 2},} \quad \alpha=\frac{x}{r}, \quad r=|x|,\end{cases}
$$


with new constants $k, s, k+1=-2 \kappa / \kappa_{1}^{\prime}=-2 \nu_{0}+4, s=(2 /(3 \gamma-1))\left(3+2 \kappa_{0} / \kappa_{1}^{\prime}\right)$ thus:

$$
k=-2 \nu_{0}+3, \quad s=-\frac{2 k}{3 \gamma-1}=-\frac{2 k}{3}
$$

Recall that $H_{0}=\left(H_{r}, H_{\Sigma}\right)$ is given in the TE (resp. TM) polarization by (122) [resp. (123)], with the conditions on $\left(\nu_{0}, n\right): \nu_{0} \leq-n-2, n>0, n \leq-\nu_{0}-2$, for TE polarization, $\nu_{0}>3 / 2$ for TM polarization.

Then $\left(p_{0}, \rho_{0}\right)$ are given by $(126,128)$ in the TE polarization, by $(129,131)$ in the TM polarization [with $v_{0}^{2}$ for $v^{2}$ in $\left.(128,131)\right]$.

\section{REFERENCES}

[1] S.I. Braginskii, Transport Phenomena in a fully ionized two-Temperatures Plasma. Zh. Eksp. Teoret. Fiziki 33 (1957) $458-472$.

[2] S.I. Braginskii, A criterion for the Applicability of the Magnetohydrodynamic Equations to a Plasma. Problems of Magnetohydrodynamics, in Proceedings of the Conference on Magnetohydrodynamics, Riga (1958).

[3] A.B. Bud'ko, F.S. Felber, A.I. Kleev, M.A. Liberman and A.L. Velikovich, Stability analysis of dynamic $Z$-pinches and thetapinches. Phys. Fluids B 1 (1989) 598-607.

[4] A.B. Bud'ko, M.A. Liberman and F.F. Kamenets, Self-similar Dynamics of dense Z-pinches, AIP Conference Proceedings 195, in Dense $Z$-pinches, Second International Conference, Laguna Beach (1989).

[5] M. Cessenat, Mathematical Methods in electromagnetism. Linear theory and applications. Series in advances Math. 41, World Scientific (1996).

[6] M. Cessenat and P. Genta, Une méthode de séparation des variables en magnétohydrodynamique. Rapport CEA-R-5714 (1996).

[7] R. Courant and K.O. Friedrichs, Mathematical Theory of Hyperbolic Flow Equations for Functions of two Variables. Interscience (1948).

[8] A.C. Eringen and G.A. Maugin, Electrodynamics of Continua. Springer (1990).

[9] D.S. Jones, Acoustic and Electromagnetic Waves. Clarendon Press (1986).

[10] K.A. Kulikovskiy and G.A. Lyubimov, Magnetohydrodynamics. Addison-Wesley (1965).

[11] L.I. Sedov, Similarity and Dimensional Methods in Mechanics (three dimensional self similar motions of compressible media). Pergamon Press (1960). 